\title{
Halting the vicious cycle within the multiple myeloma ecosystem: blocking JAM-A on bone marrow endothelial cells restores angiogenic homeostasis and suppresses tumor progression
}

\begin{abstract}
Antonio G. Solimando, ${ }^{1,2,3}$ Matteo C. Da Viá, ${ }^{1,4,5}$ Patrizia Leone, ${ }^{3}$ Paola Borrelli, ${ }^{6}$ Giorgio A. Croci, ${ }^{7,8}$ Paula Tabares, ${ }^{1,9}$ Andreas Brandl, ${ }^{1,9}$ Giuseppe Di Lernia, ${ }^{3}$ Francesco P. Bianchi, ${ }^{10}$ Silvio Tafuri, ${ }^{10}$ Torsten Steinbrunn, ${ }^{1}$ Alessandra Balduini ${ }^{11,12}$ Assunta Melaccio, ${ }^{3}$ Simona De Summa, ${ }^{13}$ Antonella Argentiero, ${ }^{2}$ Hilka Rauert-Wunderlich, ${ }^{14}$ Maria A. Frassanito, ${ }^{3}$ Paolo Ditonno, ${ }^{2}$ Erik Henke, ${ }^{15}$ Wolfram Klapper, ${ }^{7}$ Roberto Ria, ${ }^{3}$ Carolina Terragna, ${ }^{16}$ Leo Rasche,${ }^{1}$ Andreas Rosenwald ${ }^{14} \mathrm{~K}$. Martin Kortüm, ${ }^{1}$ Michele Cavo, ${ }^{16}$ Domenico Ribatti, ${ }^{17}$ Vito Racanelli, ${ }^{3}$ Hermann Einsele, ${ }^{1}$ Angelo Vacca ${ }^{3}$ and Andreas Beilhack ${ }^{1,9}$
\end{abstract}

\begin{abstract}
${ }^{1}$ Department of Medicine II, University Hospital of Würzburg, Würzburg, Germany; ${ }^{2}$ IRCCS Istituto Tumori "Giovanni Paolo II", Bari, Italy; ${ }^{3}$ Department of Biomedical Sciences and Human Oncology, Unit of Internal Medicine “Guido Baccelli", University of Bari Aldo Moro Medical School, Bari, Italy; ${ }^{4} \mathrm{Hematology}$ Unit, Fondazione IRCCS Ca' Granda Ospedale Maggiore Policlinico, Milan, Italy; ${ }^{5}$ Department of Oncology and Hemato-Oncology, University of Milan, Milan, Italy; ${ }^{6}$ Unit of Biostatistics and Clinical Epidemiology, Department of Public Health, Experimental and Forensic Medicine, University of Pavia, Pavia, Italy; ${ }^{7}$ Department of Pathology, Hematopathology Section and Lymph Node Registry, University of Kiel/University Hospital Schleswig-Holstein, Kiel, Germany; ${ }^{8}$ Pathology Unit, Department of Pathophysiology and Transplantation, University of Milan and Fondazione IRCCS, Ca' Granda, Milan, Italy; ${ }^{9}$ Interdisciplinary Center for Clinical Research Laboratory, University Hospital of Würzburg, Würzburg, Germany; ${ }^{10}$ Section of Hygiene, Department of Biomedical Science and Human Oncology, University of Aldo Moro Medical School, Bari, Italy; ${ }^{11}$ Department of Molecular Medicine, University of Pavia, Pavia, Italy; ${ }^{12}$ Department of Biomedical Engineering, Tufts University, Medford, MA, USA; ${ }^{13}$ Molecular Diagnostics and Pharmacogenetics Unit, IRCCS Istituto Tumori "Giovanni Paolo II", Bari, Italy; ${ }^{14}$ Institute of Pathology, University of Würzburg, Würzburg, Germany; ${ }^{15}$ Institute of Anatomy and Cell Biology, Julius-Maximilians Universität Würzburg, Würzburg, Germany; ${ }^{16}$ Institute of Hematology "L. and A. Seràgnoli", Bologna, Italy and ${ }^{17}$ Department of Basic Medical Sciences, Neurosciences and Sensory Organs, University of Bari Aldo Moro Medical School, Bari, Italy
\end{abstract}

\section{ABSTRACT}

Tnteractions of malignant multiple myeloma (MM) plasma cells with the microenvironment control MM plasma-cell growth, survival, drug-resistance and dissemination. As microvascular density increases in the bone marrow in MM, we investigated whether bone marrow MM endothelial cells control disease progression via the junctional adhesion molecule- $\mathrm{A}$ (JAM-A). Membrane and cytoplasmic JAM-A levels were upregulated in MM endothelial cells in 111 patients with newly diagnosed MM and in 201 with relapsed/refractory MM compared to the levels in patients with monoclonal gammopathy of undetermined significance and healthy controls. Elevated membrane expression of JAM-A on MM endothelial cells predicted poor clinical outcome. Mechanistically, addition of recombinant JAM-A to $\mathrm{MM}$ endothelial cells increased angiogenesis, whereas inhibition of this adhesion molecule impaired angiogenesis and MM growth in two-dimensional and three-dimensional in vitro cell cultures and chorioallantoic membrane assays. To corroborate these findings, we treated MM-bearing mice with a JAM-A-blocking monoclonal antibody and demonstrated impaired $\mathrm{MM}$ progression, corresponding to decreased MM-related vascularity. These findings support the concept that JAM-A is an important mediator of $\mathrm{MM}$ progression through facilitating $\mathrm{MM}$-associated angiogenesis. Elevated
Ferrata Storti Foundation

Haematologica 2021

Volume 106(7)1943-1956

\section{Correspondence:}

ANDREAS BEILHACK

beilhack_a@klinik.uni-wuerzburg.de

Received: October 7, 2019.

Accepted: April 28, 2020.

Pre-published: June 4, 2020.

https://doi.org/10.3324/haematol.2019.239913

(C)2021 Ferrata Storti Foundation

Material published in Haematologica is covered by copyright. All rights are reserved to the Ferrata Storti Foundation. Use of published material is allowed under the following terms and conditions:

https://creativecommons.org/licenses/by-nc/4.0/legalcode. Copies of published material are allowed for personal or internal use. Sharing published material for non-commercial purposes is subject to the following conditions:

https://creativecommons.org/licenses/by-nc/4.0/legalcode, sect. 3. Reproducing and sharing published material for commercial purposes is not allowed without permission in writing from the publisher. 
JAM-A expression on bone marrow endothelial cells is an independent prognostic factor for the survival of both patients with newly diagnosed MM and those with relapsed/refractory MM. Blocking JAM-A restricts angiogenesis in vitro, in utero and in vivo and represents a suitable druggable molecule to halt neo-angiogenesis and $\mathrm{MM}$ progression.

\section{Introduction}

Junctional adhesion molecule-A (JAM-A), also known as JAM-1, CD321, and F11R, belongs to the immunoglobulin superfamily. ${ }^{1}$ In healthy tissues, JAM-A regulates cell growth and differentiation, while its aberrant expression or deregulation confers a more aggressive phenotype with poor prognosis in different types of human cancers, ${ }^{1}$ including multiple myeloma (MM), ${ }^{2}$ breast, lung, brain, and head and neck cancers. ${ }^{3}$

Overactivation of JAM-A results either from upregulation or aberrant dimerization, driving the receptor in a state of constitutive signal transmission, or from excessive release of JAM-A ligands by normal and tumor cells into the microenvironment. ${ }^{4}$ Membrane-bound JAM-A and its soluble form (sJAM-A) can form homophilic interactions and also heterophilic interactions ${ }^{1}$ with lymphocyte functionassociated antigen 1 (LFA-1), afadin (AFDN), calcium/calmodulin-dependent serine protein kinase (CASK) and tight junction protein-1 (TJP1) with high receptor/ligand binding affinities. ${ }^{5}$ These interactions trigger JAM-A downstream signaling pathways involved in the regulation of tumor cell survival, growth, angiogenesis and dissemination. ${ }^{6}$

JAM-A inhibition can be achieved directly by blocking the ligand-binding site on the extracellular receptor domain with monoclonal antibodies ${ }^{7}$ and indirectly with small-molecule inhibitors. ${ }^{8}$ Moreover, neutralizing the sJAM- $\mathrm{A}^{9}$ released into the microenvironment can prevent JAM-A activation. ${ }^{10}$

JAM-A plays a pivotal role in endothelial cell physiology ${ }^{6}$ and pathology. ${ }^{2}$ Although the function of JAM-A in tumorigenesis has been investigated in solid tumors, ${ }^{3}$ and its angiogenic role has been shown in pancreatic islet carcinoma, ${ }^{11}$ data on JAM-A-related angiogenesis in hematologic neoplasms remain elusive. Since bone marrow (BM) neovascularization favors the progression of $\mathrm{MM}^{12}$ we investigated whether JAM-A can drive angiogenesis in $\mathrm{MM},{ }^{13}$ contributing to progression of the disease. ${ }^{2}$

We quantified JAM-A surface expression on BM-derived endothelial cells (MMEC) from 312 patients with MM and demonstrated that JAM-A ${ }^{\text {high }}$ MMEC correlate strongly with poor survival both in newly diagnosed (NDMM) and relapsed/refractory (RRMM) patients. Mechanistically, adding recombinant JAM-A protein to MM plasma cells (MM-cells) increased angiogenesis in both two-dimensional (2D) and three-dimensional (3D) models. Conversely, blocking JAM-A impaired MM-related angiogenesis. To corroborate these findings, we treated MM-bearing mice with JAM-A-blocking monoclonal antibodies and observed impaired MM progression and decreased MM vascularity.

\section{Methods}

\section{Patients}

Patients fulfilling the International Myeloma Working Group diagnostic criteria ${ }^{14}$ for NDMM $(n=111)$, patients with RRMM $^{15}$
( $\mathrm{n}=201)$ and subjects with monoclonal gammopathy of undetermined significance (MGUS) ( $\mathrm{n}=35$ ) were included in this study. The patients' characteristics and genetic risk stratification are provided in Online Supplementary Tables S1 and S2. The study was approved by the Ethical Committees of Bari and Würzburg University Hospitals (reference numbers 5145 and 76/13), and all patients provided informed consent to participation in the study, in accordance with the Declaration of Helsinki (details are given in the Online Supplementary Methods).

\section{Cell lines and cultures procedures}

RPMI-8226, OPM-2 and human umbilical vein endothelial cells were cultured as described elsewhere. ${ }^{3} \mathrm{MM}$-cells were cocultured with MMEC $\left(4 \times 10^{5}\right)$ at 1:1 and 1:5 cell ratios for 24 hours $(h)$ with or without an inserted transwell $(0.4 \mu \mathrm{m}$ pore size; Costar, Cambridge, MA, USA). Details are provided in the Online Supplementary Methods.

\section{Chick chorioallantoic membrane assay}

Fertilized chicken eggs were incubated at $37^{\circ} \mathrm{C}$ at a constant humidity. On day 8, sterilized gelatin sponges imbued with MMEC conditioned medium or medium obtained by treatment of MMEC with sJAM-A $(100 \mathrm{ng} / \mathrm{mL})$, with or without anti-JAMA monoclonal antibodies were implanted on the top of the chick chorioallantoic membrane (CAM) as described in more detail in the Online Supplementary Methods.

\section{Multiple myeloma xenograft mouse models}

Twenty female 8- to 10-week-old NOD.CB17Prkdcscid/NCrHsd mice (NOD-SCID; Envigo, Huntingdon, UK) were injected intratibially with $2 \times 10^{5}$ RPMI- 8226 cells suspended in phosphate-buffered saline. Mice were treated with the anti-JAM-A monoclonal antibody (Sigma-Aldrich, St. Louis, $\mathrm{MO}$, USA, mouse monoclonal clone J10.4) recognizing the distal membrane extracellular domain of JAM-A.

Twenty female 6- to 8-week-old NOD-SCID mice were injected subcutaneously (s.c.), into the right flank, with $1 \times 10^{7}$ RPMI-8226 cells suspended in $200 \mu \mathrm{L}$ RPMI-1640 medium and $200 \mu \mathrm{L}$ Matrigel ${ }^{\mathrm{TM}}$ as described previously ${ }^{16}$ and detailed in the Online Supplementary Methods.

\section{Functional in vitro assays}

Wound-healing and Matrige ${ }^{\mathrm{TM}}$ angiogenesis assays were performed as previously described and detailed in the Online Supplementary Methods.

\section{Protein expression studies and polymerase chain reaction analyses}

Western blots, enzyme-linked immunosorbent assays, human angiogenesis array and real-time reverse transcriptase polymerase chain reactions were performed according to the manufacturers' instructions (detailed in the Online Supplementary Methods).

\section{Immunohistochemistry and in silico analysis}

Details of the immunohistochemical studies and the in silico analysis, using the CoMMpass study dataset, are supplied in the Online Supplementary Methods. 


\section{Statistical analysis}

A descriptive analysis was carried out using the median and interquartile range for the quantitative variables and percentage values for the qualitative ones. The normality of the distribution of data was tested using the Shapiro-Wilk test. The levels of JAMA expression on membrane MMEC, determined by mean fluorescence intensity (MFI) in fluorescence activated cell sorting (FACS), were dichotomized into two classes, JAM-A ${ }^{\text {high }}$ and JAM-A ${ }^{\text {low }}$, choosing the median as the class boundary (Online Supplementary Methods). Moreover, for further confirmation, survival was analyzed using a model based on quartile ranges (Online Supplementary Methods).

\section{Results}

Elevated JAM-A expression on bone marrow primary multiple myeloma endothelial cells correlates with poor prognosis in both newly diagnosed and relapsed/refractory multiple myeloma

First, we compared JAM-A expression in MMEC and MGUS-derived endothelial cells (MGEC) (Figure 1A) JAM-A mRNA expression in MMEC significantly exceeded JAM-A levels in MGEC (1.8-fold difference, $P<0.0001)$ and in endothelial cells from healthy subjects (Online Supplementary Figure S1A). Subsequent western blot analy-
A
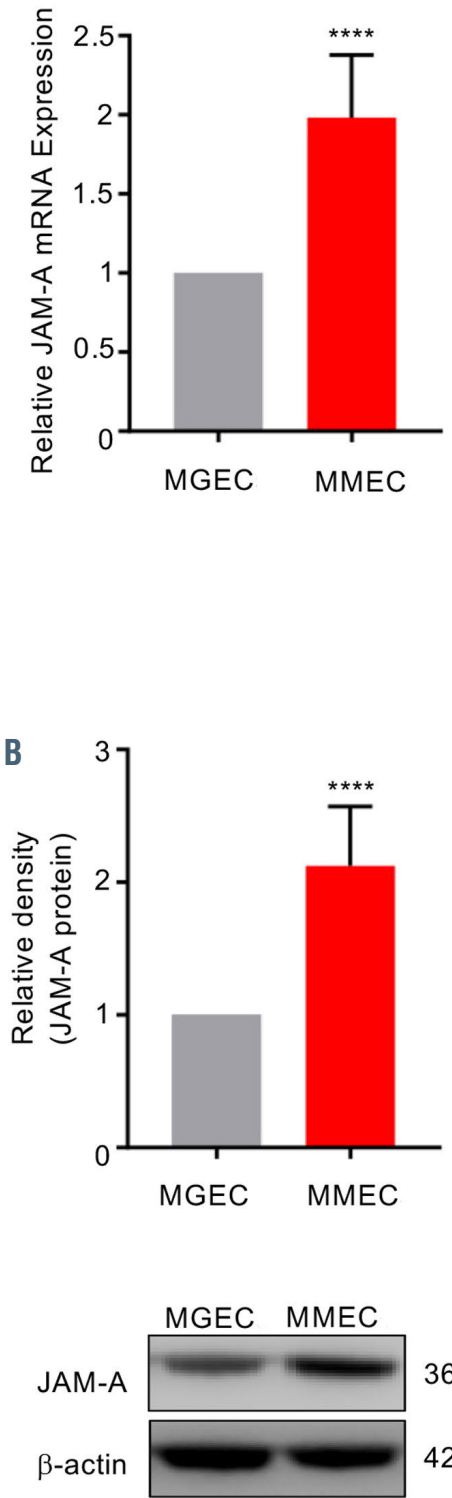

C

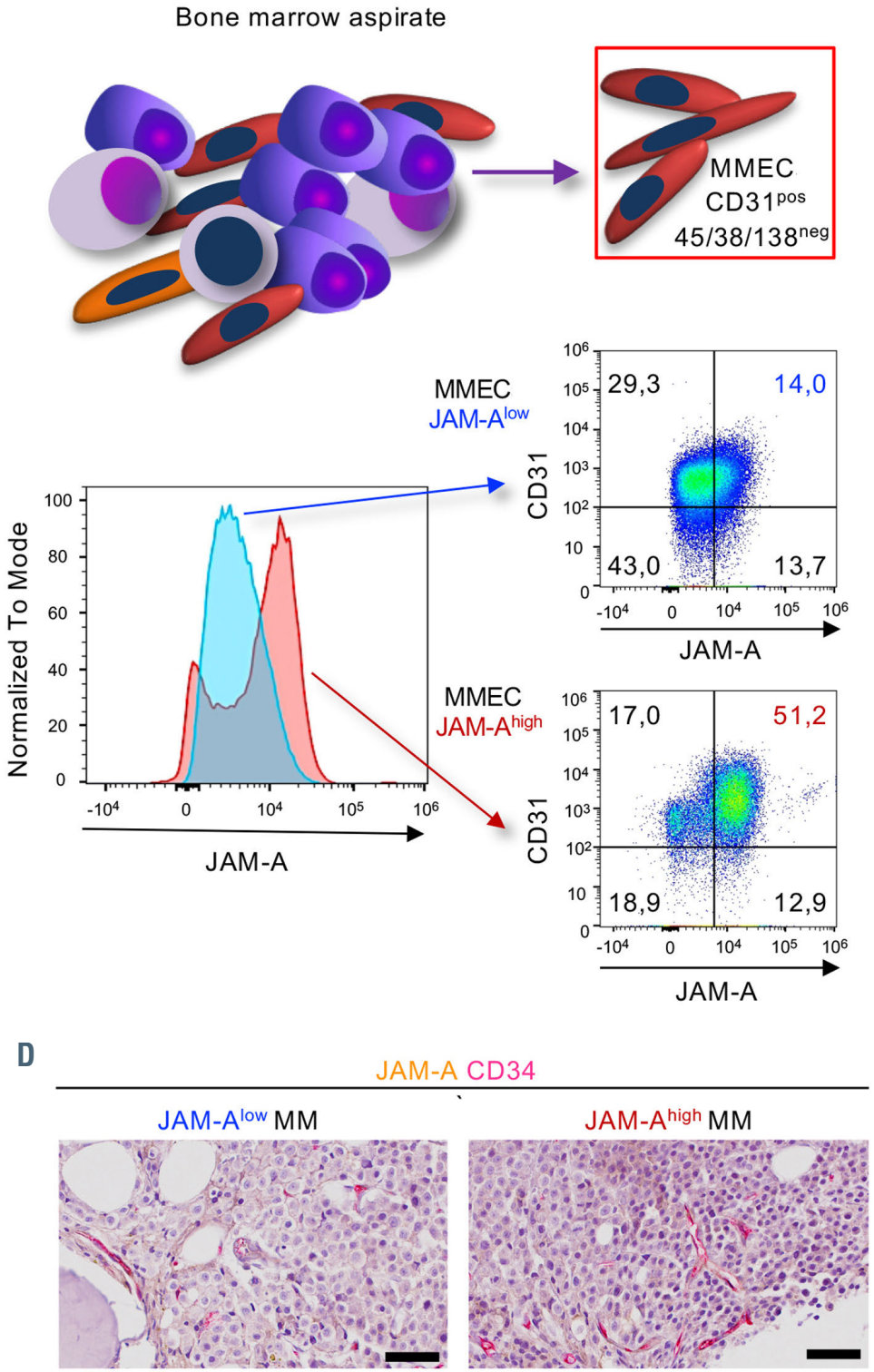

Figure 1. Elevated JAM-A expression on bone marrow primary multiple myeloma endothelial cells in newly diagnosed patients correlates with poor overall survival. (A) Relative mRNA expression level of JAM-A of endothelial cells from patients with multiple myeloma $(M M E C)(n=73)$ or monoclonal gammopathy of undetermined significance (MGEC) $(n=73)$ by real-time reverse transcriptase polymerase chain reaction. $* * * \star P<0.0001$, Mann-Whitney test. (B) Western blot densitometric analysis of basal protein expression of JAM-A of MGEC and MMEC lysates normalized to $\beta$-actin (MGEC from 24 patients with MGUS; MMEC from 24 patients with NDMM). Results are presented as mean \pm standard deviation, $* * * * P<0.0001$, Mann-Whitney test. (C) FACS analysis of JAM-A cell surface expression from representative patient-derived MMEC, identified as CD45/138/38 ${ }^{\text {neg }} / 31^{\text {pos }}$ cells. (D) JAM-A overexpression colocalizes with higher vessel density on bone marrow biopsies. Vessel density (as highlighted by CD34 [red] staining) was higher in bone marrow spaces infiltrated by JAM-A ${ }^{\text {high }}$ (brown) neoplastic plasma cells, as compared to JAM- ${ }^{\text {low }}$ cases. Magnification x 200. Scale bar $=50 \mu \mathrm{m}$. (continued on the next page) 
E

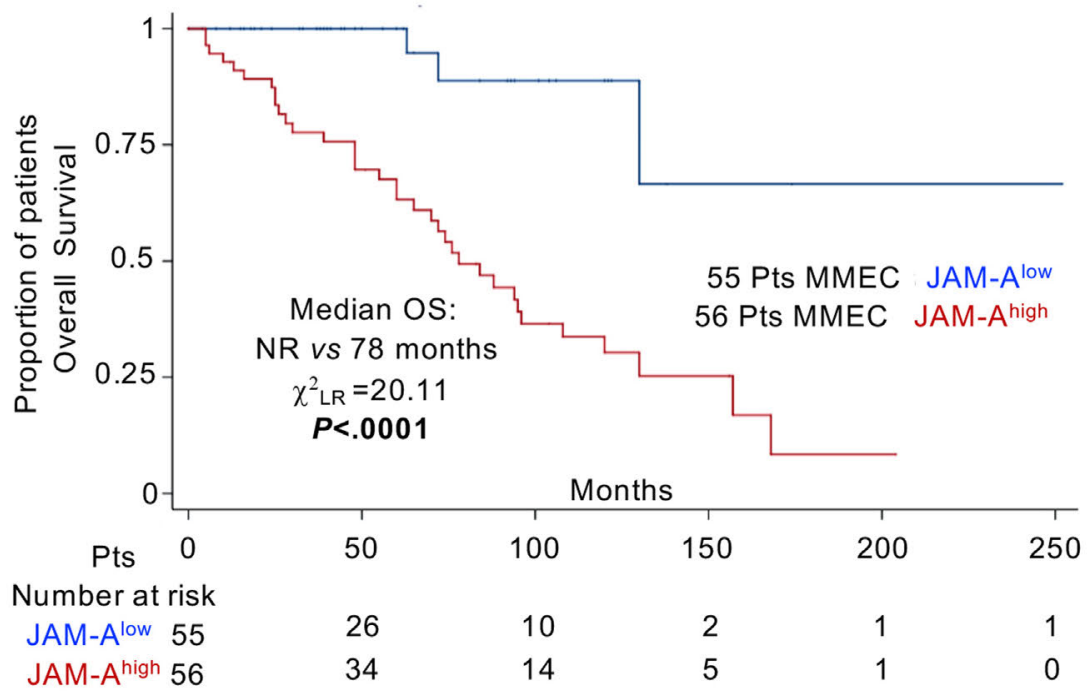

\begin{tabular}{|c|c|c|c|c|}
\hline & \multicolumn{4}{|c|}{${ }^{*}$ Cox model for Overall Survival } \\
\hline & \multicolumn{2}{|c|}{ Univariate analysis } & \multicolumn{2}{|c|}{ Multivariate analysis } \\
\hline & $\mathrm{HR}(95 \% \mathrm{Cl})$ & $P$ value & $\mathrm{HR}(95 \% \mathrm{Cl})$ & $P$ value \\
\hline $\begin{array}{l}\text { MMECs JAM-A surface } \\
\text { expression } \\
\text { (JAM-A Aigh vs JAM-Alow) }\end{array}$ & $9.14(2.80-29.76)$ & $<0.001$ & $9.11(2.79-29.76)$ & $<0.001$ \\
\hline Bone Lesion (Yes vs No) & $1.31(0.60-2.89)$ & 0.489 & - & - \\
\hline $\mathrm{Hb}(<10$ vs $>/-10 \mathrm{~g} / \mathrm{dL})$ & $1.44(0.75-2.77)$ & 0.271 & - & - \\
\hline $\begin{array}{l}\text { R-ISS } \\
\text { R-Stage I } \\
\text { R-Stage II } \\
\text { R-Stage III }\end{array}$ & $\begin{array}{c}1 \\
1.37(0.55-3.39) \\
2.11(0.76-5.86)\end{array}$ & $\begin{array}{l}0.494 \\
0.149\end{array}$ & - & - \\
\hline Sex (M vs F) & $0.58(0.29-1.13)$ & 0.110 & $0.65(0.33-1.30)$ & 0.233 \\
\hline $\begin{array}{l}\text { Chronic kidney disease } \\
\text { (Yes vs No) }\end{array}$ & $2.04(1.05-3.96)$ & 0.033 & $2.11(1.08-4.10)$ & 0.027 \\
\hline Age & $0.98(0.94-1.02)$ & 0.542 & $0.99(0.95-1.04)$ & 0.984 \\
\hline
\end{tabular}

Figure 1. (continued from the previous page). (E) Kaplan-Meier estimator of OS, by level of surface MMEC JAM-A expression. The median OS estimated in subjects with JAM-A ${ }^{\text {low }}$ MMEC cells at FACS was not reached whereas in subjects with JAM-A ${ }^{\text {high }}$ MMEC, the median OS was 78 months $(\mathrm{HR}=9.14,95 \% \mathrm{Cl}$ : 2.8-29.76, $\quad P<0.0001 ; \quad \chi_{\text {LR }}^{2}=20.11$; $P<0.0001$, upper panel). Uni- and multivariate analysis (lower panel). BM: bone marrow; MMEC: endothelial cells from patients with multiple myeloma; MGEC: endothelial cells derived from patients with monoclonal gammopathy of undetermined significance; NDMM: newly diagnosed multiple myeloma; Pts: patients. OS: overall survival; NR: not reached. R-ISS: Revised International Staging System; $\mathrm{Hb}$ : hemoglobin.

sis confirmed that JAM-A protein expression was significantly upregulated in MMEC in comparison to the levels in MGEC $(P<0.0001)$ (Figure 1B).

Because JAM-A had been previously proven to be a prominent adhesion molecule on $\mathrm{MM}$ cells, ${ }^{2}$ and is also known to form homophilic interactions, ${ }^{1}$ we investigated whether JAM-A expression in the vascular microenvironment affects disease outcome. To this end, we enrolled 312 patients, 111 with NDMM and 201 with RRMM. Employing flow cytometry on MMEC we divided the patients with NDMM on the basis of JAM-A ${ }^{\text {high }}$ and JAM-A ${ }^{\text {low }}$ MMEC surface expression (Figure 1C). Immunohistochemical analyses of BM trephines corroborated the findings (Figure 1D, Online Supplementary Figure $S 1 B$ and $C$ ). Notably, overall survival was significantly shorter in patients with JAM-A ${ }^{\text {high }}$ MMEC than in patients with JAM-A low MMEC: median not reached versus 78 months, respectively (hazards ratio $[\mathrm{HR}]=9.14$, 95\% con- fidence interval $[95 \% \quad \mathrm{CI}]: \quad 2.80-29.76 ;, \quad P<0.001$; $\chi_{\mathrm{LR}}^{2}=20.11 ; P<0.0001$ ) (Figure 1E upper panel). Strikingly, these results maintained significance also in the multivariate analysis ( $\mathrm{HR}=9.11$, 95\% CI: 2.79-29.76; $P<0.001)$ (Figure 1E lower panel). Concerning progression-free survival, only renal impairment displayed a significant impact in univariate as well as in multivariate analyses $(\mathrm{HR}=1.64$, 95\% CI: $1.09-2.47, P=0.017)$. The level of MMEC JAM-A expression did not influence risk of progression in NDMM (data not shown). Thus, JAM-A overexpression on MMEC represents a risk factor for shorter overall survival in patients with NDMM.

Next, we performed flow cytometry on samples from a cohort of 201 patients with RRMM. Within these patients, JAM- $\mathrm{A}^{\text {high }} \mathrm{MMEC}$ represented an independent poor prognostic factor for both overall survival and progression-free survival (Figure 2A and B). Survival differed significantly in patients according to the level of JAM-A expression on 
A

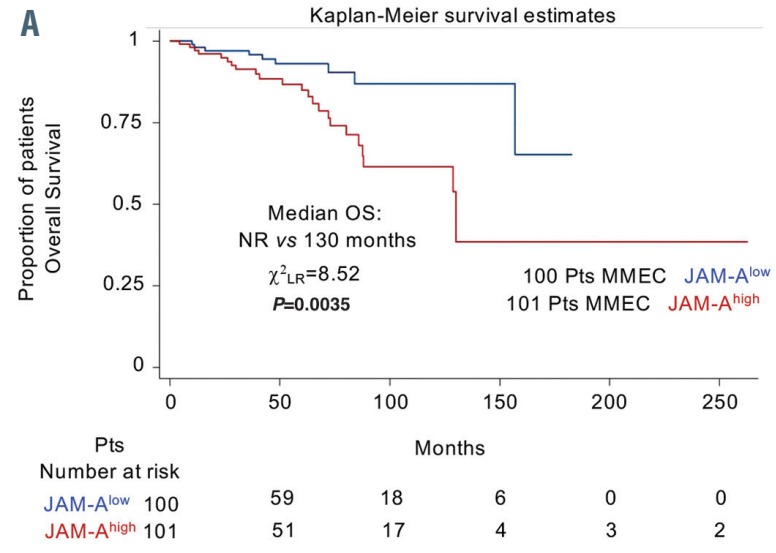

B

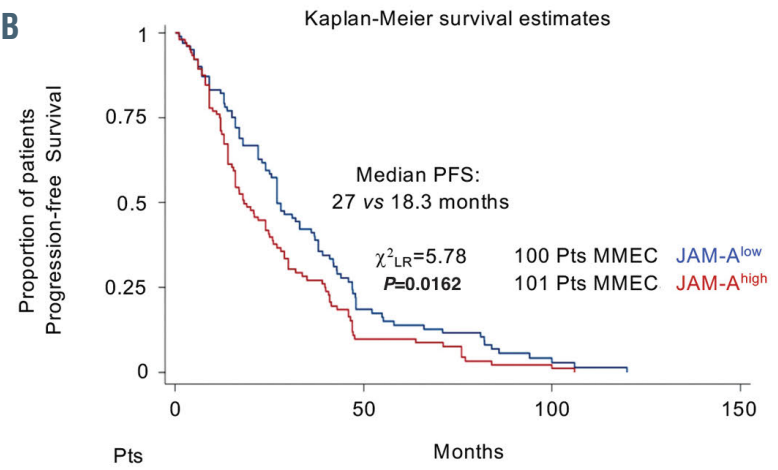

Number at risk

JAM-A low 100 JAM-A high 101

C

\begin{tabular}{|c|c|c|c|c|c|c|c|c|}
\hline & \multicolumn{4}{|c|}{${ }^{*}$ Cox model for Overall Survival } & \multicolumn{4}{|c|}{${ }^{*}$ Cox model for Progression-Free Survival } \\
\hline & Univariate an & & Multivariate a & ysis & Univariate a & & ${ }^{* *}$ Multivariate & |ysis \\
\hline & $\mathrm{HR}(95 \% \mathrm{Cl})$ & $P$ value & $\mathrm{HR}(95 \% \mathrm{Cl})$ & $P$ value & HR $(95 \% \mathrm{Cl})$ & $P$ value & $\mathrm{HR}(95 \% \mathrm{Cl})$ & $P$ value \\
\hline $\begin{array}{l}\text { MMEC JAM-A surface } \\
\text { expression } \\
\text { (JAM-A } \text { A }^{\text {high }} \text { vs JAM-A } \text { A }^{\text {low }} \text { ) }\end{array}$ & $2.96(1.37-6.37)$ & 0.006 & $2.39(1.09-5.28)$ & 0.030 & $1.41(1.05-1.88)$ & 0.019 & $1.35(1.00-1.81)$ & 0.044 \\
\hline Bone Lesion (Yes vs. No) & $1.57(0.72-3.40)$ & 0.247 & - & - & $1.09(0.81-1.48)$ & 0.546 & - & - \\
\hline Hb level (<10 vs. $210 \mathrm{~g} / \mathrm{dL}$ ) & $3.19(1.60-6.34)$ & 0.001 & - & - & $1.35(0.98-1.86)$ & 0.063 & - & - \\
\hline $\begin{array}{l}\text { R-ISS } \\
\text { R-Stage I } \\
\text { R-Stage II } \\
\text { R-Stage III }\end{array}$ & $\begin{array}{c}1 \\
7.20(1.70-30.39) \\
8.58(1.66-44.35)\end{array}$ & $\begin{array}{l}0.007 \\
0.010\end{array}$ & $\begin{array}{c}1 \\
5.34(1.24-22.97) \\
6.57(1.25-34.54)\end{array}$ & $\begin{array}{l}0.024 \\
0.026\end{array}$ & $\begin{array}{c}1 \\
1.05(0.77-1.45) \\
0.98(0.57-1.67)\end{array}$ & $\begin{array}{l}0.727 \\
0.953\end{array}$ & $\begin{array}{c}1 \\
0.96(0.68-1.33) \\
0.95(0.55-1.64)\end{array}$ & $\begin{array}{l}0.814 \\
0.866\end{array}$ \\
\hline Sex (M vs F) & $1.49(0.73-3.05)$ & 0.272 & $1.28(0.59-2.78)$ & 0.520 & $1.04(0.77-1.40)$ & 0.780 & $1.06(0.78-1.45)$ & 0.669 \\
\hline $\begin{array}{l}\text { Chronic kidney disease } \\
\text { (Yes vs. No) }\end{array}$ & $2.43(1.22-4.83)$ & 0.011 & $2.12(1.00-4.52)$ & 0.049 & $1.54(1.13-2.11)$ & 0.006 & - & - \\
\hline Age & $1.01(0.98-1.05)$ & 0.407 & $1.01(0.97-1.05)$ & 0.428 & $1.00(0.99-1.02)$ & 0.342 & $0.99(0.98-1.01)$ & 0.877 \\
\hline
\end{tabular}

Figure 2. Elevated JAM-A expression on bone marrow primary endothelial cells predicts poor prognosis in relapsed refractory multiple myeloma. Kaplan-Meier estimator of OS (A) and PFS (B), by level of surface MMEC JAM-A expression. (C) Cox model set on OS and PFS analyses. The median follow-up was 53 months (4-262 months) for OS and 23 months (1-119 months) for PFS. * Cox models adjusted for sex and age. **Cox stratified hazards regression by chronic kidney disease. OS: overall survival; PFS: progression-free survival; MMEC: bone marrow primary multiple myeloma endothelial cells. Pts: patients. NR: not reached. R-ISS: Revised International Staging System; Hb: hemoglobin.

MMEC. The median overall survival was 130 months in patients with JAM- $\mathrm{A}^{\text {high }}$ MMEC and was not reached in those with JAM-A $\mathrm{A}^{\text {low }}$ MMEC (HR=2.96, 95\% CI: $1.36-$ 6.37, $\left.P<0.006 ; \chi_{\mathrm{IR}}^{2}=8.52 ; P=0.0035\right)$ (Figure $2 \mathrm{~A}$ ).

In patients with JAM-A ${ }^{\text {low }}$ MMEC cells, the estimated median progression-free survival was 27 months, while, in subjects with JAM- $\mathrm{A}^{\text {high }}$ MMEC the median progressionfree survival was only 18.3 months ( $\mathrm{HR}=1.41,95 \% \mathrm{CI}$ : 1.05-1.88; $\left.P=0.019 ; \chi_{\mathrm{LR}}^{2}=5.78 ; P=0.0162\right)$ (Figure 2B).

Multivariate analyses confirmed that JAM-A $A^{\text {high }}$ MMEC was an independent significant risk factor for low overall survival (HR=2.39, 95\% CI: 1.09-5.28; $P=0.030)$ in much the same way that Revised International Staging System (R-ISS) stage II (HR=5.34, 95\% CI: 1.24-22.97; $P=0.024)$ and stage III disease (HR=0.57, 95\% CI: 1.25-34.54; $P=0.026)$ and chronic kidney disease $(\mathrm{HR}=2.12,95 \% \mathrm{CI}$ : $1.00-4.52 ; P=0.049$ ) were independent significant risk factors (Figure 2C). A Cox stratified model implemented for progression-free survival confirmed only high levels of membrane MMEC JAM-A as a statistically significant risk factor ( $\mathrm{HR}=1.35,95 \% \mathrm{CI}: 1.00-1.81 ; P=0.044)$ stratified by chronic kidney disease (Figure 2C).

Interestingly, only JAM-A ${ }^{\text {high }}$ MMEC remained significant in the multivariate model. Moreover, we found a significant association in the setting of RRMM between JAM-A ${ }^{\text {high }}$ MMEC and R-ISS stage II and III disease $\left(\chi^{2}=17.4, P<0.0001\right)$ and the risk of extramedullary dis- semination $\left(\chi^{2}=7.04, P=0.008\right)$. Thus, JAM-A surface expression on BM endothelial cells derived from MM patients exerted a strong and independent effect, with a linear trajectory, on overall survival in both cohorts and an additive poor prognostic impact on progression-free survival in the RRMM cohort. Additionally, we divided MMEC JAM-A surface expression of the entire cohort (Online Supplementary Figure S1D) into quartiles (JAM$\mathrm{A}^{\mathrm{Q} 1}$ to JAM- $\mathrm{A}^{\mathrm{Q}}$ ) and then compared the outcomes of patients in the lowest quartile (JAM-A $A^{01}$ ) to those of patients in the highest quartile (JAM-A ${ }^{\text {Q4 }}$ ). Strikingly, overall survival differed significantly: the median overall survival was 88 months in JAM- $A^{04}$ patients and was not reached in JAM- ${ }^{\mathrm{Q} 1}$ patients $(\mathrm{HR}=8.24,95 \%$ CI: 3.2 $\left.20.9, P<0.0001 ; \chi_{\mathrm{LR}}^{2}=28.15 ; P<0.0001\right)$. Interestingly, JAM-A ${ }^{04}$ MMEC maintained significance in the multivariate Cox-model ( $\mathrm{HR}=6.36,95 \%$ CI: 2.30-17.63; $P<0.001)$. This comparison further corroborated the role of JAM-A-positive vs. JAM-A-negative MMEC in predicting poor clinical outcome in our cohort (Online Supplementary Figure S1E, upper and lower panels). The absence of a statistically significant impact on progression-free survival of patients with NDMM is likely due to a more pronounced effect of JAM-A MMEC expression in more advanced stages of MM. This suggests the importance of JAM-A within the BM microenvironment during disease progression. 

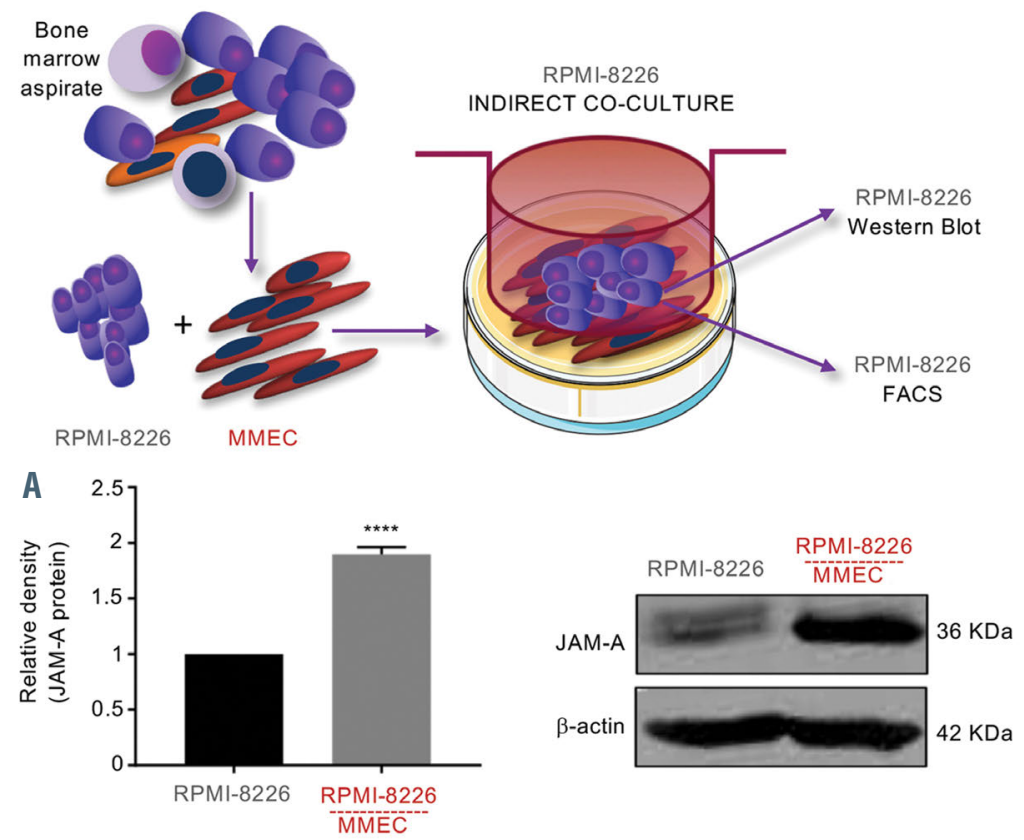

B

RPMI-8226 INDIRECT CO-CULTURE
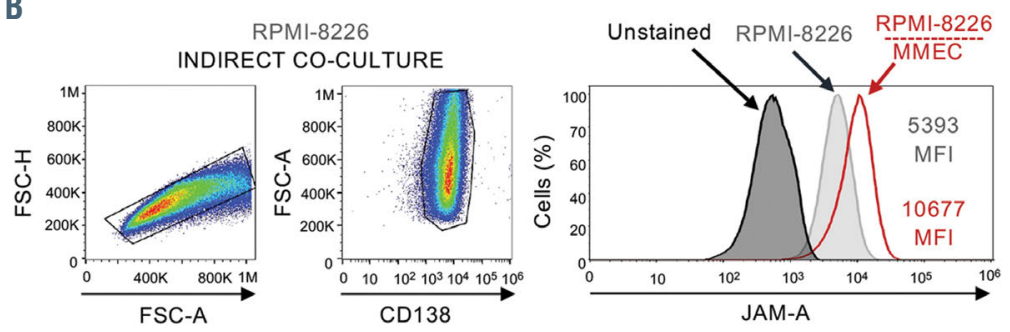

Figure 3. Bone marrow primary multiple myeloma endothelial cells enhance JAM-A expression on multiple myeloma cells. (A, B) Experimental design depicted at the top. RPMI-8226 cells were cultured alone or cocultured with MMEC at a 1:5 ratio (RPMI8266:MMEC) in inserted transwells and analyzed for JAM-A expression by western blotting (A) and flow cytometry (B). (C) Experimental design shown at top, RPMI-8226 cells were maintained for $24 \mathrm{~h}$ in CM from MMEC or MGEC. Cells were harvested and lysed and the extracted proteins immunoblotted for JAM-A expression. Overall densitometric analyses are reported. (D) RPMI-8226 cells were also analyzed by FACS after culture for $24 \mathrm{~h}$ in MGEC or MMEC CM. Results are presented as mean \pm standard deviation (MGEC from 24 patients with MGUS; MMEC from 24 patients with NDMM), $* * * * P<0.0001$, MannWhitney test. BM: bone marrow; MMEC: endothelial cells from patients with multiple myeloma; MGEC: endothelial cells derived from patients with monoclonal gammopathy of undetermined significance; NDMM: newly diagnosed $\mathrm{MM}$; $\mathrm{CM}$ : conditioned medium: MFI: mean fluorescence intensity.

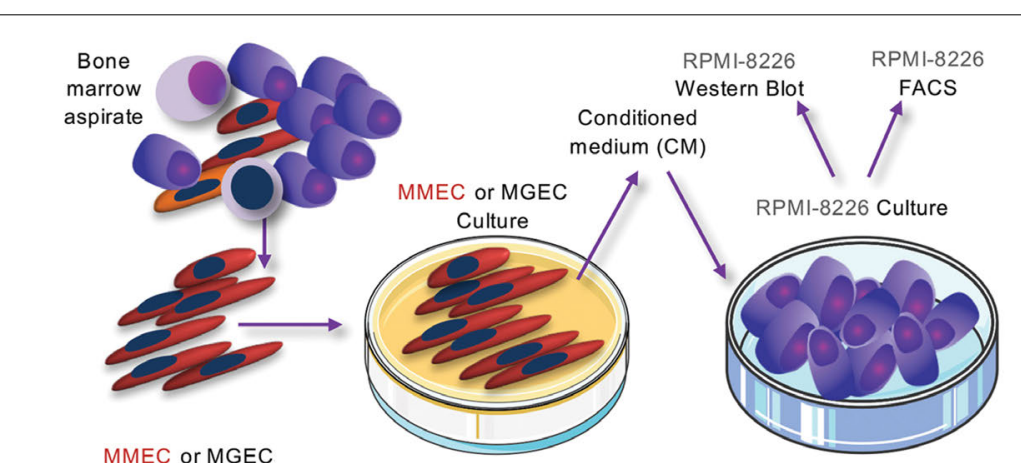

C
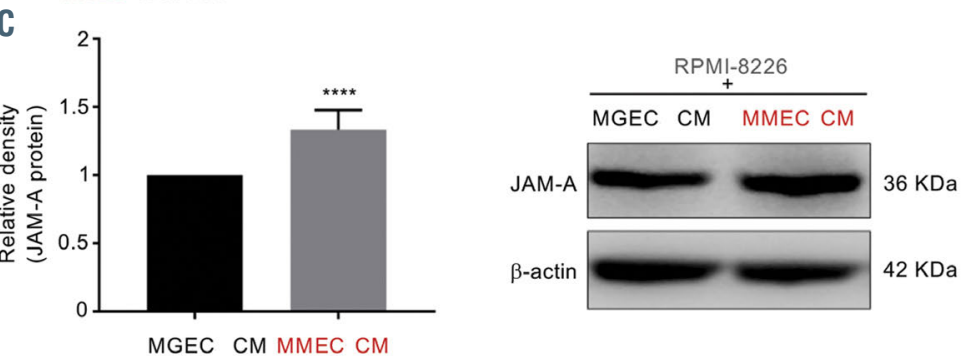

D

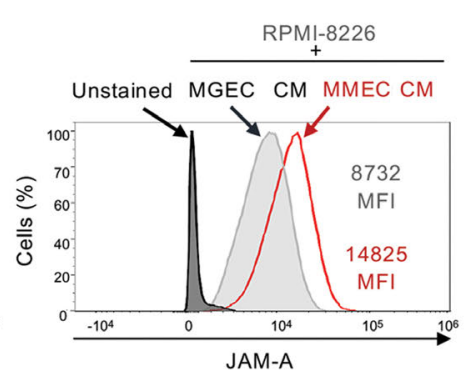


A
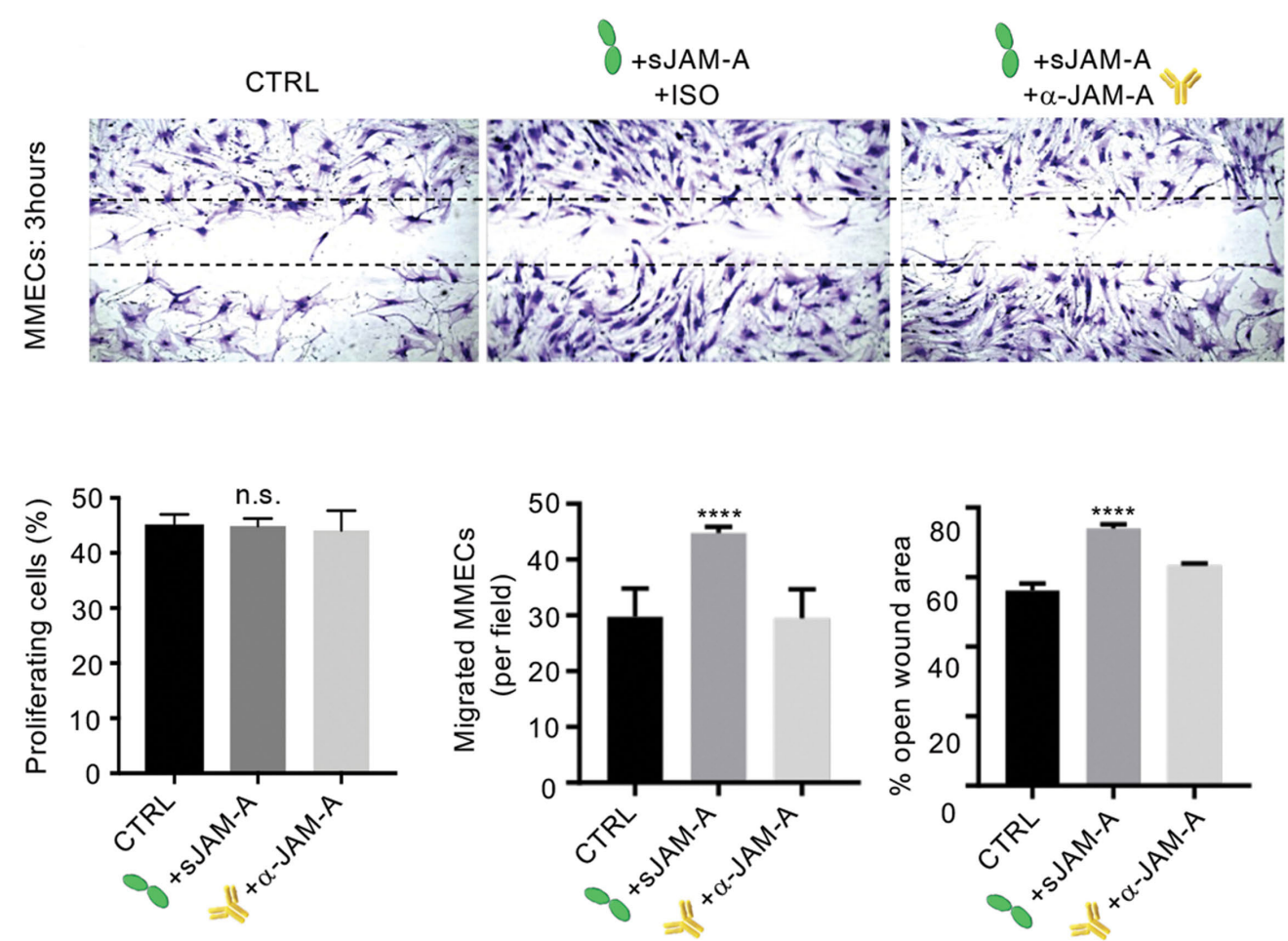

B
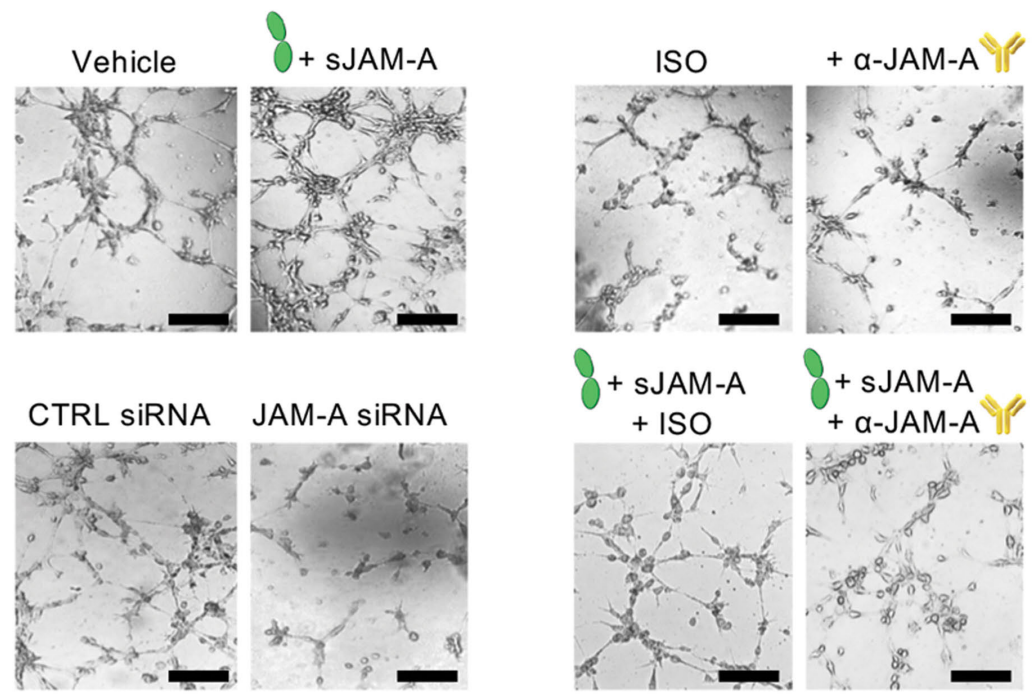

Branching points fold change
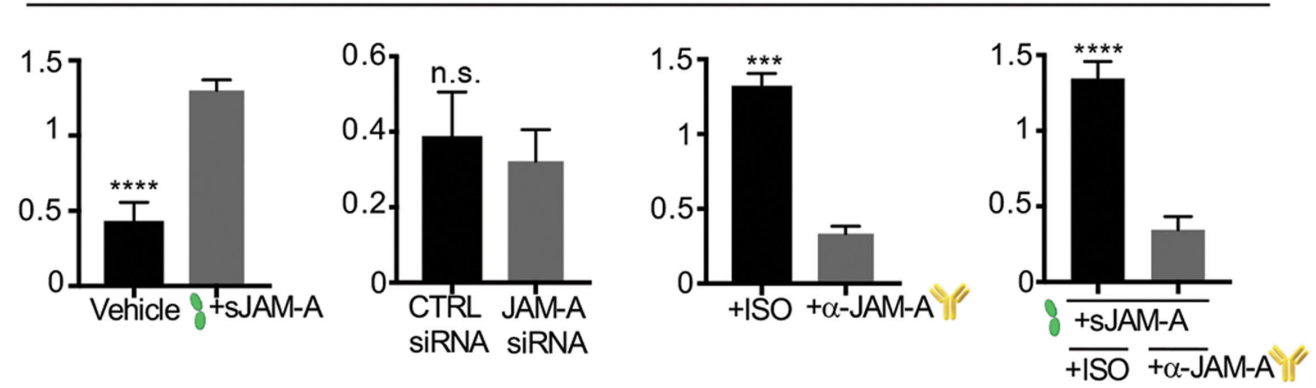

Figure 4. Legend on next page. 


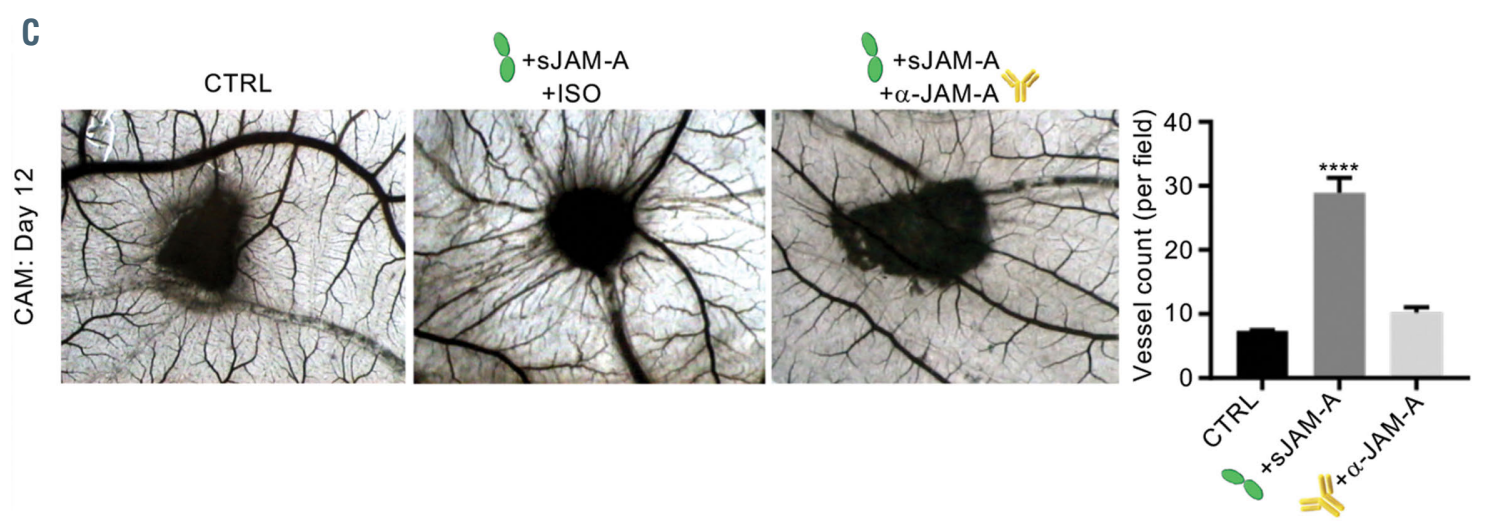

D

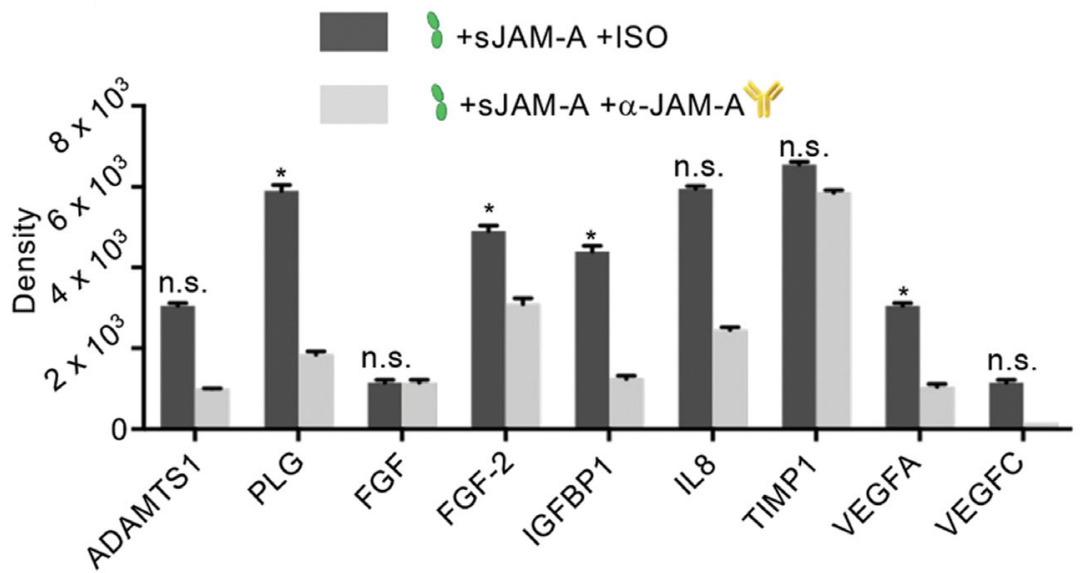

Figure 4. Pivotal role of JAM-A in multiple myeloma-associated angiogenesis in two- and three-dimensional conditions. (A) Upper panel. Confluent monolayers of MMEC underwent a scratch wound-healing assay. Three hours after scratching photographs were taken of MMEC that had been maintained in MMEC CM alone (CTRL) or previously supplemented for $12 \mathrm{~h}$ with SJAM-A at $100 \mathrm{ng} / \mathrm{mL}$ and treated with isotype control (+SJAM-A +ISO) or anti-JAM-A (+SJAM-A + $\alpha-J A M-A)$ blocking antibody. Lower panel: Migrating cells in each wound were counted. Counts of proliferating and migrating cells of six independent experiments. $\star \star \star \star P<0.0001$, Mann-Whitney test. (B) Photographs at 3 hours of newly-formed capillary networks after MMEC were seeded on a Matrigel ${ }^{T M}$ layer. Direct comparison of MMEC in CM vs. MMEC treated with $100 \mathrm{ng} / \mathrm{mL}$ sJAM-A (upper left panel). Independent experiment to assess JAM-A inhibition in MMEC treated with an anti-JAM-A blocking antibody or isotype control antibody (upper right panel). Independent experiment to assess JAM-A knock-down in MMEC comparing treatment with JAM-A specific siRNA vs. non-specific scrambled siRNA without addition of SJAM-A (lower left panel). Independent experiment to assess the effect of blocking JAM-A after addition of 100 $\mathrm{ng} / \mathrm{mL}$ of SJAM-A by comparing capillary formation after MMEC treatment with sJAM-A and anti-JAM-A blocking antibody vs. sJAM-A and isotype control antibody (lower right quadrant). Representative pictures of three biological replicates. Skeletonization of the meshes were analyzed and branching points measured. Data are normalized to control. Scale bar=100 $\mu \mathrm{m}$. (C) Chorioallantoic membrane assay with the gelatin sponge loaded with MMEC CM alone (CTRL) or with MMEC CM supplemented with SJAM-A (+SJAM-A), in the presence or absence of $0.5 \mu \mathrm{g} / \mathrm{mL}$ anti-JAM-A monoclonal antibody. On day 12 , pictures were taken in ovo. One representative experiment is shown at 50X magnification. Newly formed vessels were counted. Mann-Whitney test. (D) An array of 55 human angiogenesis-related proteins was performed on MMEC CM after SJAM-A treatment without and with blocking with the anti-JAM-A monoclonal antibody. Array spots were analyzed with Image Lab v. 1.51 software and normalized to positive control signal intensities. Graph bars represent the pixel density of the detected angiogenesis-related cytokines in two independent experiments. Values are expressed as mean \pm standard deviation of ten independent experiments. Mann-Whitney test. $* P<0.05$; $* * * * P<0.0001$, versus SFM as control. See Online Supplementary Figure S3 and the main text for more details. MMEC: bone marrow primary multiple myeloma endothelial cells; $\alpha$-JAM-A: monoclonal antibody against JAM-A; SJAM-A: soluble JAM-A; CM: conditioned medium; CTRL: control; SFM: serum-free medium; n.s.: not significant. CAM: chick chorioallantoic membrane. ADAMTS1: human metalloproteinase with thrombospondin type 1 motifs; PLG: plasminogen; FGF-2: fibroblast growth factor-2; IGFBP1: insulinlike growth factor binding protein 1; IL8: interleukin-8; TIMP1: tissue inhibitor matrix metalloprotease 1; VEGFA: vascular endothelial growth factor A; VEGFC: vascular endothelial growth factor $\mathrm{C}$.

\section{Multiple myeloma endothelial cells enhance JAM-A expression on multiple myeloma plasma cells}

To address how interactions with MMEC functionally influence MM-cell biology, we performed indirect and direct co-culture experiments of MMEC with MM-cell lines. JAM-A expression levels increased on MM-cells when co-cultured with MMEC (Figure $3 \mathrm{~A}$ and $\mathrm{B}$ ). We next exposed MM-cells to primary MMEC- or MGEC-derived culture media. JAM-A protein expression was higher on MM-cells after exposure to MMEC medium than after exposure to MGEC medium (Figure $3 \mathrm{C}$ and D, respectively). Results confirmed JAM-A upregulation upon direct coculture experiments (Online Supplementary Figure S2A). Notably, only after direct co-culture did MMEC recapitulate the same behavior as that of MM-cells (Online
Supplementary Figure S2B). Consistently, sJAM-A levels also increased after co-culture of MM-cells with MMEC (Online Supplementary Figure S2C). Similarly to RPMI-8226 cells, OPM-2 cells upregulated JAM-A after direct co-culture with MMEC (Online Supplementary Figure S2D), but not after indirect culture (data not shown). These data indicate that both cell-cell contact and soluble factors released by MMEC into the BM microenvironment upregulated JAM-A expression on MM-cells. MMEC JAM-A upregulation parallels this dynamic process, suggesting a vicious cycle, promoting MM growth by supporting angiogenesis.

\section{JAM-A enhances angiogenesis in two- and three-dimensional conditions}

We hypothesized that JAM-A upregulation during the 


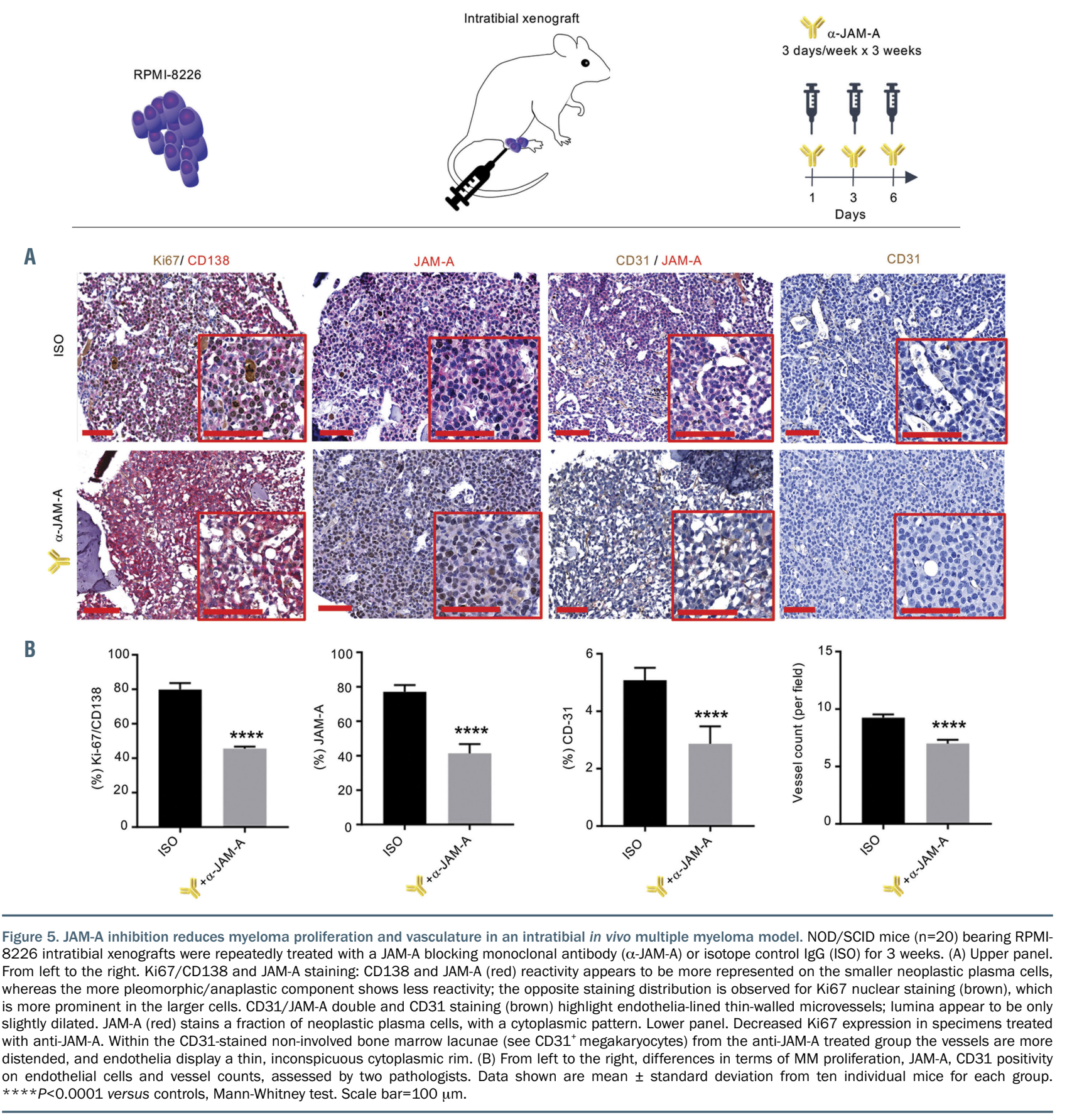

progression of MM may enhance angiogenesis. To study this, we treated MMEC with increasing concentrations of human recombinant sJAM-A and measured different parameters of angiogenesis. ${ }^{17,18}$ To examine whether JAMA directly affects spontaneous MMEC migration, we performed experiments in two-dimensional (2D) and threedimensional (3D) environments. Enhanced spontaneous MMEC migration was observed after $12 \mathrm{~h}$ of sJAM-A treatment in a $2 \mathrm{D}$ scratch assay in which migrating MMEC were counted (Figure 4A, upper and lower panels). Blocking JAM-A abolished the enhanced MMEC migration (Figure 4A, upper panel) and reduced the numbers of migrating MMEC (Figure 4A, lower panel). In a 2D angiogenesis assay, sJAM-A treatment increased endothelial structural complexity in terms of branching points and vessel length, which are parameters of angiogenesis. Three hours after seeding, sJAM-A treatment resulted in a structured capillary network, while the control remained in a rudimentary stage of organization with small clumps of cells distributed on the Matrigel ${ }^{\mathrm{TM}}$ layer (Figure 4B, upper left quadrant). Furthermore, we tested the effect of JAM-A inhibition in MMEC by both siRNA and an antibody blocking JAM-A without adding sJAM-A (Figure 4B, lower left and upper right quadrants, respectively). Consistently, blocking JAM-A with a monoclonal antibody impaired the capillary network formation and resulted in poorly skeletonized structures (Figure $4 \mathrm{~B}$, lower right quadrant). The observed down-modulation of MMEC migration, reduced number of branching points and shorter vessel length occurred independently of cytotoxic 

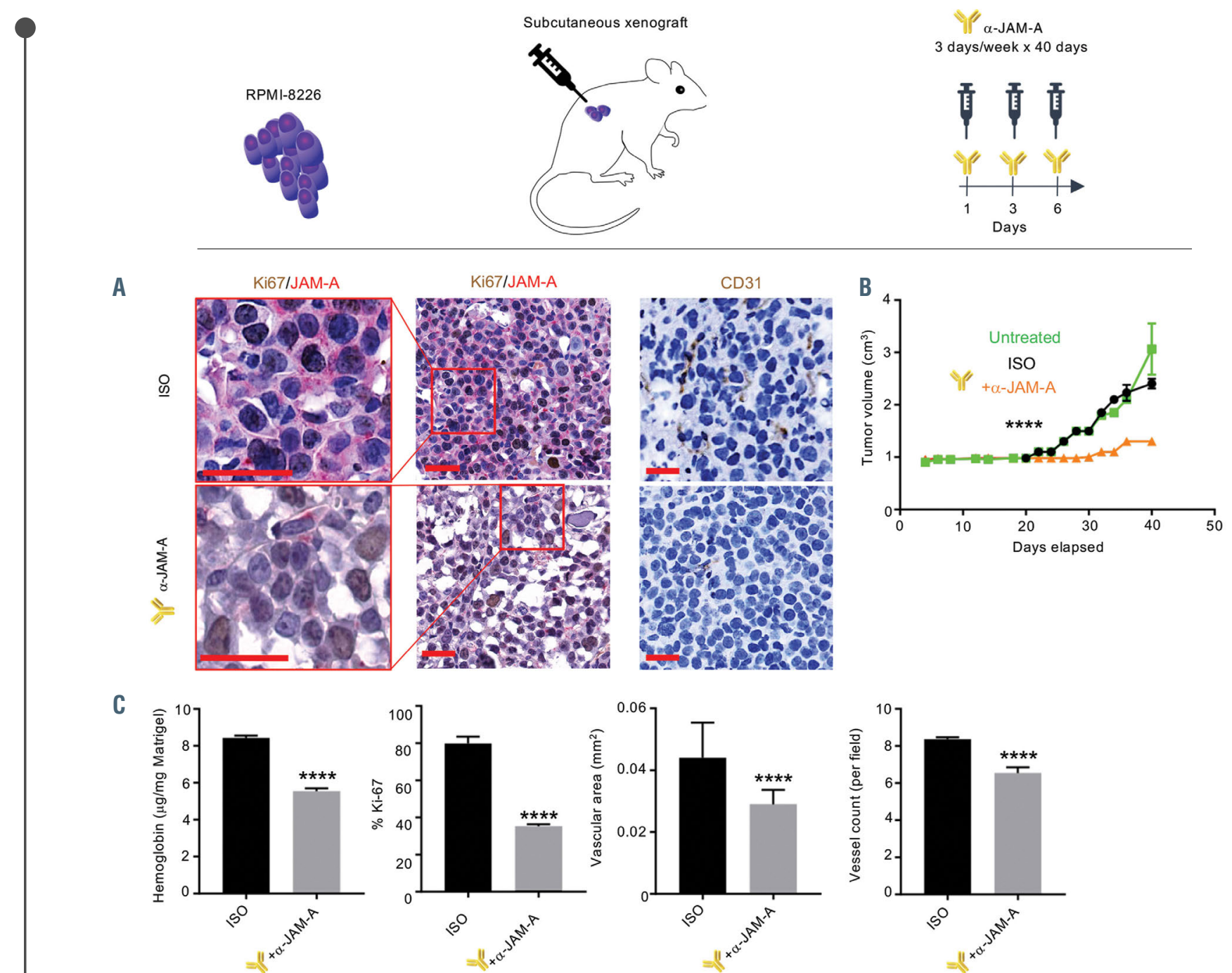

Figure 6. JAM-A inhibition restricts angiogenesis and tumor growth in subcutaneous multiple myeloma xenograft model. NOD/SCID mice ( $\mathrm{n}=20$ ) bearing RPMI-8226 subcutaneous xenografts were repetitiously treated with a JAM-A blocking monoclonal antibody ( $\alpha$-JAM-A), or isotope control IgG (ISO) or with vehicle only for 40 days for 3 days/week. (A) Immunohistochemistry staining: JAM-A (red) reactivity is stronger in the smaller neoplastic cells whereas it is lower in the more pleomorphic/anaplastic components. The opposite staining distribution is observed for Ki67 nuclear staining (brown), which is more clear-cut in the larger cells. CD31 staining shows focal positivity in the control group and is absent in the group treated with the JAM-A blocking antibody. (B) Treatment was continued for 3 days/week for 40 days and tumor volumes were measured every 2 days with a caliper. (C) Hemoglobin values, Ki-67 positivity, vessel area and number of vessels expressed as mean \pm standard deviation of three independent experiments. ${ }^{*} P<0.05$ versus vehicle-treated control. Scale bar $=50 \mu \mathrm{m} . * * * * P<0.0001$ versus controls; Mann-Whitney test.

effects, since JAM-A neutralization did not affect MMEC survival (Online Supplementary Figure S3A, left and right panels).

Based on the 2D observations, we investigated whether JAM-A could influence structured MM-associated angiogenesis in a 3D CAM assay. CAM were implanted with gelatin sponges imbued with either MMEC conditioned medium as the control (CTRL) or MMEC conditioned medium with sJAM-A (+SJAM-A), in the presence or absence of a monoclonal antibody blocking JAM-A. MMEC conditioned medium stimulated new vessel formation in CAM, ${ }^{19}$ and this effect was markedly enhanced by the addition of sJAM-A. The effect could be selectively inhibited by treatment with a sJAM-A blocking antibody as treatment with a cocktail containing an isotype IgG1 control antibody (sJAM-A + ISO, middle panel) did not reduce vessel formation (Figure $4 \mathrm{C}$ ).

To explore potential factors that enhance JAM-A-mediated $\mathrm{MM}$ angiogenesis, we compared conditioned media from MMEC supplemented with sJAM-A before and after treatment with the JAM-A blocking antibody with an angiogenesis array (Figure 4D, Online Supplementary Figure $S 3 B)$. sJAM-A strongly reduced anti-angiogenic and increased pro-angiogenic factors secreted by MMEC, ${ }^{16}$ such as plasminogen (PLG), fibroblast growth factor 2 (FGF-2), insulin-like growth factor binding protein 1 (IGFBP1) and vascular endothelial growth factors A and C (VEGFA, VEGFC). Reverse transcriptase polymerase chain reaction analysis corroborated the proteomic findings and revealed sJAM-A-induced transcriptional upregulation of these factors and ligands (PLG and ENO1, JAM-A with LFA-1 and TJP1) (Online Supplementary Figure S3C-L). Moreover, because JAM-A can form homophilic interactions with JAM-A itself1 as well as heterophilic interactions with LFA-1, TJP1, CAV1 and CASK, we investigated whether the expression of these ligands correlated with MM-MMEC interactions. Direct co-culture of RPMI-8226 cells and MMEC significantly increased LFA-1 and CAV1 
A

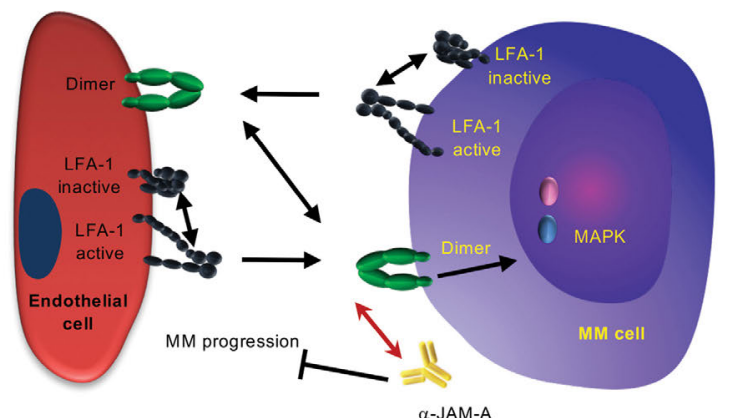

C

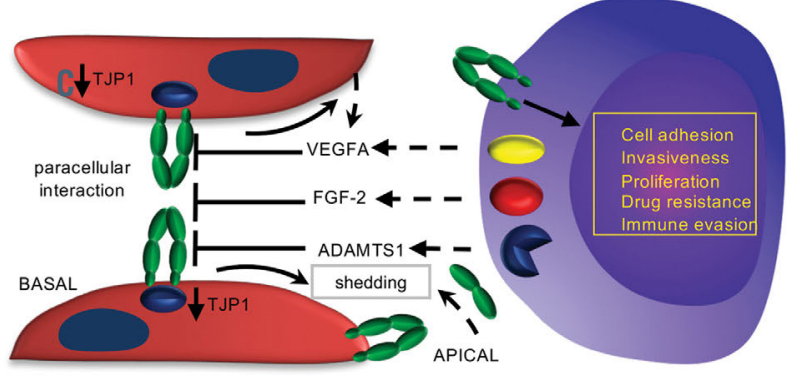

B

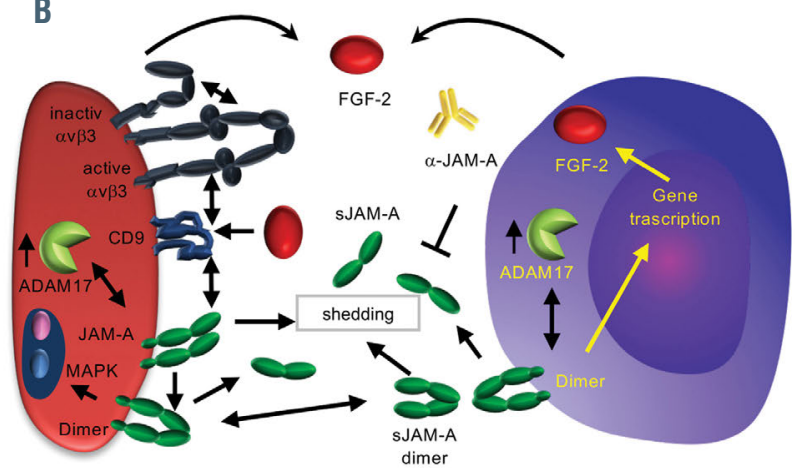

D

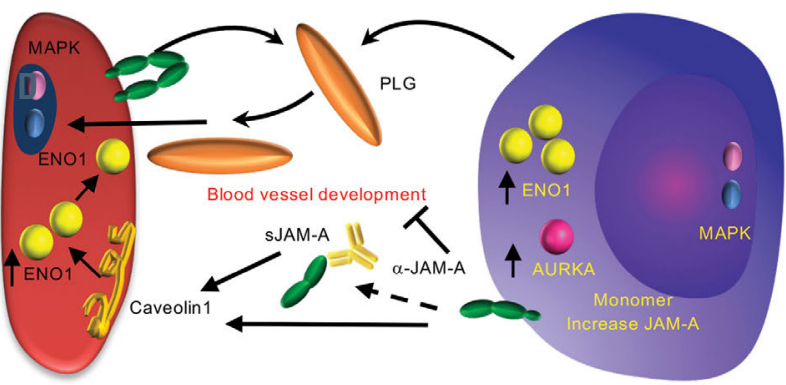

Figure 7. JAM-A boosts multiple myeloma-related angiogenesis in the bone marrow microenvironment. (A) Molecular interactions between MM plasma cells and MMEC: cell-adhesion mediated changes via trans-homo/heterophilic JAM-A interactions. (B) FGF-2 mediates the pro-angiogenic and proliferative roles of JAM-A and the release of monomeric JAM-A from the ternary complex through an unknown mechanism. We speculate that once monomeric JAM-A is available at a membrane level, it forms homodimers that mediate downstream signaling and is also susceptible for cleavage and shedding via ADAM17. (C) JAM-A-mediated cytoskeleton rearrangement via TJP1 downregulation and cell function modification. Depending on myeloma cell-mediated interactions, the endothelial cells can lose their tight junction and thus vascular permeability can increase. (D) An interaction between PLG and ENO1 triggers angiogenesis and microenvironmental modifications via JAM-A. Caveolin1 modulates the transport of cytosolic ENO-1 to the cell surface. $\alpha$-JAM-A: monoclonal antibody against JAM-A. BM: bone marrow; MMEC: bone marrow primary multiple myeloma endothelial cells; FGF-2: fibroblast growth factor-2; ADAM17: ADAM metallopeptidase domain 17; TJP1: tight junction protein-1; PLG: plasminogen; ENO1: enolase 1; LFA-1: lymphocyte function-associated antigen 1; MAPK: mitogen-activated protein kinase; $\alpha v \beta 3$ : integrin alpha $V$ beta 3; CD9: CD9 molecule; VEGFA: vascular endothelial growth factor A; ADAMTS1: human metalloproteinase with thrombospondin type 1 motifs. AURKA: aurora kinase A.

on MM-cells, whereas TJP1, CASK and ADAM17 expression levels decreased (Online Supplementary Figure S3G-K). We therefore investigated whether the induced gene expression was non-random, by studying other molecules involved in neoplastic angiogenesis processes, ${ }^{12}$ namely VEGFA, VEGFC, HGF, FGF ${ }^{16}$ and Aurora kinase A (AURKA); ${ }^{20}$ also in this case we found significant VEGFA and AURKA gene upregulation after MM-MMEC co-culture (Online Supplementary Figure S3F and L).

These data support the concept that MM-MMEC interactions enhance angiogenesis. Thus, we investigated whether MM cells participate actively in the angiogenesis program in a reciprocal interaction with the BM microenvironment in patients and whether a pro-angiogenic gene signature could identify patients with worse progressionfree and overall survival. We therefore studied 646 NDMM patients enrolled in the CoMMpass trial, comparing two different cohorts, based on survival outcome (alive vs. dead for overall survival and progressed vs. ongoing disease for progression-free survival) performing a supervised analysis based on the gene expression of the pro-angiogenic factors contained in the angiogenesis array and other well-known JAM-A interactors. Strikingly, these two cohorts differed significantly: JAM-A, ENO-1, VEGFA and $A U R K A$ were all overexpressed in patients experiencing shorter progression-free and overall survival. Conversely, reduced TJP1 expression in patients correlated with poor survival (Online Supplementary Table S3). These gene expression data confirmed the protein expression results from our cohort of patients. Exogenous JAM-A modulated the secretory profile of MMEC, favoring angiogenesis, and highlighted the tight connection with an angiogenic environment, comprising key angiogenic factors, such as PLG, FGF-2, IGFBP1, VEGFA and VEGFC.

\section{JAM-A inhibition impairs angiogenesis and inhibits tumor growth in vivo}

To investigate whether JAM-A inhibition may affect in vivo angiogenesis and in turn MM-cell growth, we employed two different mouse models. To mimic advanced $\mathrm{MM}^{21}$ we injected RPMI-8226 cells intratibially into NOD-SCID mice and analyzed bone specimens after anti-JAM-A treatment. ${ }^{2}$ Blocking JAM-A reduced MM-cell proliferation and angiogenesis (Figure $5 \mathrm{~A}$ ). The difference was statistically significant regarding numbers and percentages of $\mathrm{Ki}-67^{\text {high }}$ proliferating $\mathrm{MM}$-cells $(79.87 \pm 1.242$ and $35.38 \pm 0.3455$ in the groups treated with isotype control and anti-JAM-A, respectively; $P<0.0001)$ and vessels $/ \mathrm{mm}^{2}$ field (9.3 and 7.1 in the groups treated with isotype control and anti-JAM-A, respectively $P<0.0001)$ (Figure $5 \mathrm{~B}$ ). The group treated with the antibody against JAM-A expressed lower JAM-A levels $(79.78 \pm 1.443$ and $36.98 \pm 0.466$ in the groups treated with isotype control and anti-JAM-A, respectively; $P<0.0001)$, lower CD31\% (5.58 \pm 1.34 and $3.48 \pm 0.646$ in the groups treated with isotype control and anti-JAM-A, respectively; $P<0.0001)$ and 
lower vessel density (calculated as number of vessels $/ \mathrm{mm}^{2}$ ) than the group treated with the isotype control, which had a higher number of vessels with well-lit lumina $(9.77 \pm 2,63$ and $6.48 \pm 0,631$ in the groups treated with isotype control vs. anti-JAM-A, respectively; $P<0.0001$ ).

To assess the activity of the anti-JAM-A blocking antibody on angiogenesis on a solitary plasmacytoma in vivo and to monitor MM-cell growth at extra-osseous sites noninvasively with a caliper, we employed a subcutaneous $\mathrm{MM}$ xenograft model. This approach enables dissection of the endothelial bystander effect on BM-independent extramedullary $\mathrm{MM}^{21}$ Thus, we employed a second in vivo xenograft model engrafting RPMI-8226 cells subcutaneously into the flanks of NOD/SCID mice. ${ }^{21}$ The animals were randomized at day 3 after engraftment and treated with either anti-JAM-A monoclonal antibody or a non-specific isotype control antibody intraperitoneally for 3 days/week for 40 days. Subsequently, we measured the vascular area, tumor volume and hemoglobin content of the MM mass. Blocking JAM-A reduced the vascular area in the soft tissue MM masses compared to that in animals treated with an isotype control (difference between medians $-0.015 ; P<0.0001$ ). No adverse events occurred upon continuous anti-JAM-A treatment.

Notably, after 40 days, the vascular area increased significantly in tumors and MM disease progressed more in controls than in the group treated with the monoclonal antibody against JAM-A (Figure 6A, CD31 staining, and 6B). In isotype-treated control mice, tumors grew exponentially, contrasting with the only limited tumor growth in antiJAM-A-treated animals (Figure 6B, Online Supplementary Figure S4A). Lower hemoglobin content confirmed poor MM vascularization in the anti-JAM-A-treated mice (8.4 \pm 0.04 in the isotype-treated control mice vs. $5.5 \pm 0.04$ in the anti-JAM-A-treated group; $P<0.0001,95 \%$ CI: -3.02 to -2.8 (Figure 6C). Ki-67-staining, vascular area and vessel count confirmed that blocking JAM-A strongly reduced $M M$ vascularity and disease progression. Furthermore, JAM-A blocking significantly reduced pro-angiogenic factors such as FGF-2 and VEGF-A in the peripheral blood of MM-bearing mice (Online Supplementary Figure S4B-D).

\section{Discussion}

Angiogenic switching is a key process during transition from premalignant asymptomatic MGUS to full-blown $\mathrm{MM}$. Angiogenic parameters in the BM at the time of diagnosis were widely considered to be able to predict MM progression..$^{22}$ In solid tumors, such as breast, lung, head and neck, and brain cancers, JAM-A activation promotes tumor progression, while its inhibition by anti-JAM- $\mathrm{A}^{2}$ agents reduces tumor growth. ${ }^{11}$ We demonstrated in four independent experimental settings that JAM-A essentially stimulates $\mathrm{MM}$-associated angiogenesis. In the CAM assay, a monoclonal antibody neutralizing JAM-A caused a strong reduction of the number of vessels, implying that JAM-A exerts an essential angiogenic stimulus that could not be replaced by any other compensating factor contained in the MMEC conditioned medium. ${ }^{23}$ Our new findings pinpoint JAM-A as an attractive target in MM patients.

JAM-A appears pivotal in MM evolution, which can be explained by several angiogenic mechanisms. ${ }^{24,25}$ First, we demonstrated significantly higher JAM-A levels on MMEC from NDMM patients than on MGEC. Furthermore, we could link the high JAM-A surface expression on MMEC with a significantly shorter overall survival in both NDMM and RRMM and, at even more advanced disease stages, higher JAM-A expression levels also correlated with reduced progression-free survival. We therefore examined the pathophysiological basis responsible for favoring MM progression. As already described for the HGF/cMET axis, ${ }^{26,27} \mathrm{JAM}$-A acts within the BM microenvironment, sustaining the neoplastic clone and promoting MM-related angiogenesis both directly and indirectly by priming MMEC. Thus, JAM-A and its soluble isoform SJAM-A appear to feed into a vicious cycle involving MMEC, generating a malignant environment favorable for MM progression. Although JAM-A is expressed in several solid cancers, ${ }^{3}$ to our knowledge this is the first report of the role of endothelial JAM-A expression in the MM tumor microenvironment. Homophilic interactions between recombinant sJAM-A and membrane JAM-A have been demonstrated biochemically. ${ }^{10}$ Homophilic JAM-A interactions can be inhibited by an anti-JAM-A monoclonal antibody that binds to an epitope close to the $\mathrm{N}$-terminus of the mature protein $^{10}$ as well as by a peptide that corresponds to the $\mathrm{N}$ terminal 23 residues of the mature protein. ${ }^{28}$ This suggests that the homophilic trans-interaction is mediated through the membrane-distal V-type Ig-like JAM-A domain at the $\mathrm{N}$-terminus of the molecule. Targeting this domain of the JAM-A molecule on MMEC in our in vitro co-culture systems suggested that this type of interaction mediates the MM-MMEC crosstalk. In line with previous reports about MMEC sustaining MM growth, 29,30 our disease models showed that during the transition from the pre-angiogenic to the angiogenic phase, proliferation of tumor cells and neovascularization intensely involve over-expression of JAM-A on MMEC. MMEC were responsive to the presence of sJAM-A in the surrounding microenvironment, which increased their JAM-A surface protein expression. sJAM-A directly and indirectly upregulated JAM-A on the bystander MM-cells, independently of their basal JAM-A expression status. These observations support the concept that cellular components of MM BM, including MMEC, can release JAM-A to sustain disease progression and prepare a tumor-"friendly" niche, exerting significant modulation on FGF-2, VEGF-A and PLG/ENO1 downstream effects.

JAM-A has been described to interact with CD9, a wellknown driver of MM-related drug resistance ${ }^{31}$ and clinical prognosis. ${ }^{32}$ We found significant expression of FGF-2, a potent stabilizer and activator of a ternary complex involving JAM-A, CD9 and $\alpha v \beta 3$ integrin, a novel potential therapeutic target. ${ }^{33}$ Peddibhotla et al. described that the aggregation of this ternary complex can activate downstream pathway cascades to induce proliferation, migration and an angiogenic stimulus to endothelial cells. ${ }^{34}$ Our in silico validation shed more light on this pathophysiological process. ENO1 encodes $\alpha$-enolase, which in the cytoplasm works as a plasminogen receptor and has been described to show upregulated membrane expression in several types of cancer. $^{35,36}$ Of note, plasminogen upregulation had been correlated with tumor invasion and angiogenesis ${ }^{37}$ its activation, derived from the interaction with $\alpha$-enolase, prompted activation of downstream signaling such as the MEK-ERK pathway, which was able to promote cell invasion and angiogenesis further. $\alpha$-enolase can also modulate antitumor immune responses. Cappello et al. described that $\alpha$ enolase $^{\text {high }}$ myeloid-derived suppressor cells could not 
adhere to tumor necrosis factor- $\alpha$-primed endothelial cells in the presence of an anti-ENO-1 monoclonal antibody. Consequently, decreased migration of the myeloid-derived suppressor cells reduced in situ immunosuppression, enhancing T-cell-mediated immunity against malignant cells. . $^{8,39}$

We found that JAM-A overexpression in MMEC strongly correlated with the expression of ADAMTS1, a regulator of angiogenesis ${ }^{40}$ and immune-surveillance, ${ }^{41}$ which appears to play a central role in preparing a favorable BM milieu. ${ }^{42} \mathrm{We}$ also identified that the expression of JAM-A on the surface of MMEC was inversely correlated with ADAM17 expression. Conversely, sJAM-A release correlated directly with ADAM17 upregulation, a mechanism described for endothelial cells in inflammation. ${ }^{9}$ ADAM17 upregulation has also been observed in MM in the context of fractalkine release, ${ }^{43}$ which identifies this system as a potential novel therapeutic target in $\mathrm{MM}$ patients to disrupt a vicious circle enhancing the MM niche.

JAM-A levels also correlated strongly with AURKA levels in $\mathrm{MM}$ patients. This finding may link JAM-A-mediated cell adhesion to $\mathrm{MM}$ resilience and drug resistance. ${ }^{20}$ Indeed, proteasome engulfment-derived proteotoxicity ${ }^{44}$ and invasiveness through epithelial-mesenchymal-transition and cell adhesion ${ }^{45}$ are complex biological events that affect prognosis. ${ }^{46} \mathrm{We}$ also demonstrated that the interaction between MMEC and MM-cells affects the expression of JAM-A and other fundamental molecules, such as TJP1 and LFA-1. This reciprocal "education" parallels the invasive behavior of the MM-cells towards the endothelial counterpart, instructing the vasculature to interact actively with the malignant cells, potentially driving their survival and drug resistance. ${ }^{47,48}$ In line with this, increased JAM-A endothelial levels correlated strongly with unfavorable and resistant MM stages such as high R-ISS disease stages and the risk of extramedullary development.

A network of interactions between JAM-A and $\alpha$-enolase $^{49}$ emphasizes the strong communication with the MM niche environment to allow persistence and sustaining proliferative signaling. Therefore, JAM-A may represent a key factor in the nurturing substrate ${ }^{50}$ supporting the evolution of MM. Moschetta et al. ${ }^{13}$ previously described the interderpendency of endothelium and MM-cells: endothelial progenitor cell trafficking enhances MM progression, particularly at an early disease stage. Rajkumar et al. highlighted a progressive increase in BM angiogenesis along the spectrum of plasma cell disorders from MGUS to advanced MM. ${ }^{12}$ Integrating the prognostic relevance of JAM-A expressed by MMEC and our experimental data led us to propose JAM$\mathrm{A}$ as a key player in coordinating the interactions with the $\mathrm{MM}$ milieu enabling a permissive BM ecosystem during the aggressive disease evolution from NDMM to RRMM.

Indeed, the anti-MM effect of blocking JAM-A also relies on a complex antiangiogenic effect, especially in critical transition phases of MM progression, such as the passage from MGUS to symptomatic MM and from responsive to drug-refractory disease, interfering with a main proangiogenic factor and with MM-cell proliferation. Our 2D and 3D models showed that, mechanistically, JAM-A drives $\mathrm{MM}$-associated angiogenesis via a homophilic interaction and through identified downstream targets, namely FGF2, VEGF-A and PLG/ENO1, in the BM microenvironment. Moreover, the clinical impact demonstrated in a large cohort of consecutive individuals pinpoints the JAM-A axis as a new player in $\mathrm{MM}$-associated angiogenesis able to refine the prognostic stratification of patients, especially those with more advanced disease.

The close link between MM and the BM microenvironment appears paradigmatic for MM evolution and disease progression. We connected the interaction of MMEC with $\mathrm{MM}$-cells via the adhesion molecule JAM-A. Our data point towards a vicious cycle of JAM-A overexpression on MMEC reflected by a higher JAM-A expression on the tumoral counterpart. Shed from the cell surface, sJAM-A enhances the establishment of homophilic JAM-A complexes fostering $\mathrm{MM}$ niche formation. Finally, our results may lead to the development of JAM-A-based therapeutic strategies directed against $M M$-interactions with the tumor microenvironment (Figure 7A-D). Clearly, these findings need to be confirmed in a larger population of patients in a carefully designed, prospective, clinical study.

\section{Disclosures}

No conflicts of interest to disclosure.

\section{Contributions}

$A G S, M C D V, V R, H E, A V$ and $A B e$ designed and performed research, analyzed and interpreted data and wrote the manuscript; AGS, MCDV, PL, GC, PTG, GDL, ABr, EH, HR-W, $D R$ performed research and analyzed data; $A G S, M C D V, A A$, $P B, G C, F P B, A M, S D S, T S, A B a, M A F, W K, A R$ and $S T$ analyzed data; $A G S, H E, R R, L R, P D, K M K, V R$ and $A V$ provided patients' samples; AGS, MCDV, CT, MC, VR, AV, HE and $A B e$ designed research, interpreted data, and edited the manuscript; all authors revised the manuscript and approved its submission. VR and HE contributed equally to this study. $A V$ and $A B e$ contributed equally to the conception and design of this study.

\section{Acknowledgments}

The authors acknowledge the Multiple Myeloma Research Foundation for providing an updated and comprehensive real-life $M M$ dataset for the international scientific community. The in silico analysis and the relative clinical correlation were generated as part of the Multiple Myeloma Research Foundation Personalized Medicine Initiative. We thank the members of the laboratories of Beilhack and Vacca for lively discussions. We also thank Drs. Julia Delgado Tascón, Claudia Siverino and Marco Metzger, and Mss Katharina Schmiedgen, Charlotte Botz-Von Drathen, Hannah Manz and Vittoria Musci for technical support and valuable discussions. Public consulted datasets are available at https://research.mmrf.org, CoMMpass longitudinal, prospective observational study (release IA12).

\section{Funding}

This work was supported by the Bayerische Forschungsstiftung consortium FortiTher (WP2TP3), the Deutsche Forschungsgemeinschaft $\mu$ Bone consortium (2084/1, 401253051), by the Italian Association for Cancer Research (AIRC) through an Investigator Grant (n. 20441 to VR), by the GLOBALDOC Project - CUP H96J17000160002 approved with A.D. n. 9 from Puglia Region, financed under the Action Plan for Cohesion approved with Commission decision C (2016) 1417 to AGS. This research project was supported in part by the Apulian Regional Project "Medicina di Precisione" to AGS. AR was supported by the Wilhelm Sander-Stiftung (grant $n$. 2014.903.1). 


\section{References}

1. Steinbacher T, Kummer D, Ebnet K. Junctional adhesion molecule-A: functional diversity through molecular promiscuity. Cell Mol Life Sci. 2018;75(8):1393-1409.

2. Solimando AG, Brandl A, Mattenheimer K, et al. JAM-A as a prognostic factor and new therapeutic target in multiple myeloma. Leukemia. 2018;32(3):736-743.

3. Leech AO, Cruz RGB, Hill ADK, Hopkins AM. Paradigms lost-an emerging role for over-expression of tight junction adhesion proteins in cancer pathogenesis. Ann Transl Med. 2015;3(13):184.

4. Severson EA, Parkos CA. Mechanisms of outside-in signaling at the tight junction by junctional adhesion molecule A. Ann N Y Acad Sci. 2009;1165:10-18

5. Ebnet K, Schulz CU, Meyer Zu Brickwedde MK, Pendl GG, Vestweber D. Junctional adhesion molecule interacts with the PDZ domain-containing proteins AF-6 and ZO-1. J Biol Chem. 2000;275(36):27979-27988.

6. Ebnet K. Junctional adhesion molecules (JAMs): cell adhesion receptors with pleiotropic functions in cell physiology and development. Physiol Rev. 2017;97(4): 1529-1554.

7. Terral G, Champion T, Debaene F, et al. Epitope characterization of anti-JAM-A antibodies using orthogonal mass spectrometry and surface plasmon resonance approaches. mAbs. 2017;9(8):1317-1326.

8. Vellanki SH, Cruz RGB, Jahns H, et al. Natural compound tetrocarcin-A downregulates junctional adhesion molecule-A in conjunction with HER2 and inhibitor of apoptosis proteins and inhibits tumor cell growth. Cancer Lett. 2019;440-441:23-34.

9. Koenen RR, Pruessmeyer J, Soehnlein O, et al. Regulated release and functional modulation of junctional adhesion molecule $\mathrm{A}$ by disintegrin metalloproteinases. Blood. 2009;113(19):4799-4809.

10. Bazzoni G, Martinez-Estrada OM, Orsenigo F, Cordenonsi M, Citi S, Dejana E. Interaction of junctional adhesion molecule with the tight junction components ZO-1, cingulin, and occludin. I Biol Chem. 2000;275(27):20520-20526.

11. Murakami M, Francavilla C, Torselli I, et al. Inactivation of junctional adhesion molecule-A enhances antitumoral immune response by promoting dendritic cell and $\mathrm{T}$ lymphocyte infiltration. Cancer Res. 2010;70(5):1759-1765.

12. Rajkumar SV, Mesa RA, Fonseca R, et al. Bone marrow angiogenesis in 400 patients with monoclonal gammopathy of undetermined significance, multiple myeloma, and primary amyloidosis. Clin Cancer Res. 2002;8(7):2210-2216.

13. Moschetta M, Mishima Y, Kawano Y, et al. Targeting vasculogenesis to prevent progression in multiple myeloma. Leukemia. 2016;30(5):1103-1115

14. Rajkumar SV, Dimopoulos MA, Palumbo A, et al. International Myeloma Working Group updated criteria for the diagnosis of multiple myeloma. Lancet Oncol. 2014;15(12):e538-548.

15. Rajkumar SV, Harousseau J-L, Durie B, et al. Consensus recommendations for the uniform reporting of clinical trials: report of the International Myeloma Workshop Consensus Panel 1. Blood. 2011; 117(18):4691-4695

16. Rao L, De Veirman K, Giannico D, et al. Targeting angiogenesis in multiple myeloma by the VEGF and HGF blocking DARPin ${ }^{\circledR}$ protein MP0250: a preclinical study. Oncotarget. 2018;9(17):13366-13381

17. Jridi I, Catacchio I, Majdoub H, et al. The small subunit of Hemilipin2, a new heterodimeric phospholipase A2 from Hemiscorpius lepturus scorpion venom, mediates the antiangiogenic effect of the whole protein. Toxicon. 2017;126:38-46.

18. Nowak-Sliwinska P, Alitalo K, Allen E, et al. Consensus guidelines for the use and interpretation of angiogenesis assays. Angiogenesis. 2018;21(3):425-532.

19. Ribatti D, Nico B, Vacca A, Presta M. The gelatin sponge-chorioallantoic membrane assay. Nat Protoc. 2006;1(1):85-91.

20. Oin Y, Zhang S, Deng S, et al. Epigenetic silencing of miR-137 induces drug resistance and chromosomal instability by targeting AURKA in multiple myeloma. Leukemia. 2017;31(5):1123-1135.

21. Mitsiades CS, Anderson KC, Carrasco DR. Mouse models of human myeloma. Hematol Oncol Clin North Am. 2007; 21(6):1051-1069, viii.

22. Rajkumar SV, Leong T, Roche PC, et al. Prognostic value of bone marrow angiogenesis in multiple myeloma. Clin Cancer Res. 2000;6(8):3111-3116

23. Ria R, Catacchio I, Berardi S, et al. HIF-1 $\alpha$ of bone marrow endothelial cells implies relapse and drug resistance in patients with multiple myeloma and may act as a therapeutic target. Clin Cancer Res. 2014;20(4): 847-858.

24. Fukuhara T, Kim J, Hokaiwado S, et al. A novel immunotoxin reveals a new role for CD321 in endothelial cells. PloS One. 2017; 12(10): $\mathrm{e} 0181502$

25. Stamatovic SM, Sladojevic N, Keep RF, Andjelkovic AV. Relocalization of junctional adhesion molecule A during inflammatory stimulation of brain endothelial cells. Mol Cell Biol. 2012;32(17):3414-3427.

26. Moschetta M, Basile A, Ferrucci A, et al. Novel targeting of phospho-cMET overcomes drug resistance and induces antitumor activity in multiple myeloma. Clin Cancer Res. 2013:19(16):4371-4382

27. Ferrucci A, Moschetta M, Frassanito MA, et al. A HGF/cMET autocrine loop is operative in multiple myeloma bone marrow endothelial cells and may represent a novel therapeutic target. Clin Cancer Res. 2014:20(22):5796-5807.

28. Babinska A, Kedees MH, Athar H, et al. F11receptor (F11R/JAM) mediates platelet adhesion to endothelial cells: role in inflammatory thrombosis. Thromb Haemost. 2002;88(5):843-850

29. Cooke VG, Naik MU, Naik UP. Fibroblast growth factor-2 failed to induce angiogenesis in junctional adhesion molecule-A-deficient mice. Arterioscler Thromb Vasc Biol. 2006;26(9):2005-2011.

30. Leone P, Di Lernia G, Solimando AG, et al. Bone marrow endothelial cells sustain a tumor-specific CD8+ T cell subset with suppressive function in myeloma patients. Oncoimmunology. 2019;8(1):e1486949.

31. Hu X, Xuan H, Du H, Jiang H, Huang J. Down-regulation of CD9 by methylation decreased bortezomib sensitivity in multiple myeloma. PloS One. 2014;9(5):e95765.

32. De Bruyne E, Bos TJ, Asosingh $\mathrm{K}$, et al. Epigenetic silencing of the tetraspanin CD9 during disease progression in multiple myeloma cells and correlation with survival. Clin Cancer Res. 2008;14(10):29182926

33. Wallstabe L, Mades A, Frenz S, Einsele H, Rader C, Hudecek M. CAR T cells targeting $\alpha v \beta 3$ integrin are effective against advanced cancer in preclinical models. Adv Cell Gene Ther. 2018;1(2):e11.
34. Peddibhotla SSD, Brinkmann BF, Kummer $\mathrm{D}$, et al. Tetraspanin CD9 links junctional adhesion molecule-A to $\alpha v \beta 3$ integrin to mediate basic fibroblast growth factor-specific angiogenic signaling. Mol Biol Cell 2013;24(7):933-944

35. Tu S-H, Chang C-C, Chen C-S, et al. Increased expression of enolase alpha in human breast cancer confers tamoxifen resistance in human breast cancer cells. Breast Cancer Res Treat. 2010;121(3):539 553.

36. Yu Y-Q, Wang L, Jin Y, et al. Identification of serologic biomarkers for predicting microvascular invasion in hepatocellular carcinoma. Oncotarget. 2016;7(13):1636216371.

37. Rossignol P, Ho-Tin-Noé B, Vranckx R, et al. Protease nexin-1 inhibits plasminogen activation-induced apoptosis of adherent cells. J Biol Chem. 2004;279(11):10346-10356.

38. Cappello P, Tonoli E, Curto R, Giordano D, Giovarelli M, Novelli F. Anti- $\alpha$-enolase antibody limits the invasion of myeloid-derived suppressor cells and attenuates their restraining effector $\mathrm{T}$ cell response. Oncoimmunology. 2016;5(5):e1112940.

39. Castella B, Foglietta M, Riganti C, Massaia M. Vy9V82 T cells in the bone marrow of myeloma patients: a paradigm of microenvironment-induced immune suppression. Front Immunol. 2018;9:1492.

40. Casal C, Torres-Collado AX, Plaza-Calonge $\mathrm{MDC}$, et al. ADAMTS1 contributes to the acquisition of an endothelial-like phenotype in plastic tumor cells. Cancer Res. 2010;70(11):4676-4686.

41. Hope C, Foulcer S, Jagodinsky J, et al. Immunoregulatory roles of versican proteolysis in the myeloma microenvironment. Blood. 2016;128(5):680-685

42. Li J, Zou K, Yu L, et al. MicroRNA-140 inhibits the epithelial-mesenchymal transition and metastasis in colorectal cancer. Mol Ther Nucleic Acids. 2018;10:426-437.

43. Marchica V, Toscani D, Corcione A, et al Bone marrow CX3CL1/fractalkine is a new player of the pro-angiogenic microenvironment in multiple myeloma patients. Cancers. 2019;11(3):321.

44. Savitski MM, Zinn N, Faelth-Savitski M, et al. Multiplexed proteome dynamics profiling reveals mechanisms controlling protein homeostasis. Cell. 2018;173(1):260-274.e25.

45. Ren B-J, Zhou Z-W, Zhu D-J, et al. Alisertib induces cell cycle arrest, apoptosis, autophagy and suppresses EMT in HT29 and Caco-2 cells. Int $\mathrm{J}$ Mol Sci. 2015;17(1):41.

46. Noll JE, Vandyke K, Hewett DR, et al PTTG1 expression is associated with hyperproliferative disease and poor prognosis in multiple myeloma. I Hematol Oncol. 2015;8:106

47. Waldschmidt JM, Simon A, Wider D, et al. CXCL12 and CXCR7 are relevant targets to reverse cell adhesion-mediated drug resistance in multiple myeloma. Br J Haematol. 2017;179(1):36-49.

48. Zhang X-D, Baladandayuthapani V, Lin $\mathrm{H}$, et al. Tight junction protein 1 modulates proteasome capacity and proteasome inhibitor sensitivity in multiple myeloma via EGFR/JAK1/STAT3 signaling. Cancer Cell. 2016;29(5):639-652.

49. Ramroop JR, Stein MN, Drake JM. Impact of phosphoproteomics in the era of precision medicine for prostate cancer. Front Oncol. 2018;8:28.

50. Mundy GR. Metastasis to bone: causes, consequences and therapeutic opportunities. Nat Rev Cancer. 2002;2(8):584-593. 